\title{
Equações diferenciais impulsivas com retardamento: soluções oscilatórias e soluções periódicas
}

\author{
Marta Cilene Gadotti
}

Orientador: Prof. Dr. Plácido Zoega Táboas

Tese apresentada ao lnstituto de Ciências Matemáticas e de Computação - ICMC-USP. como parte dos requisitos para obtenção do titulo de Doutor em Ciências - Matemática.

USP - São Carlos

Junho de 2002 


\section{Sumário}

$\begin{array}{ll}\text { Introdução } & 1\end{array}$

1 Preliminares 3

2 Estudo sobre Soluções Oscilatórias $\quad 9$

2.1 Introdução . . . . . . . . . . . . . . . . . . . . . . . . 9

2.2 Um problema impulsivo escalar e não-linear . . . . . . . . . . 12

2.3 Alguns resultados para un problema planar . . . . . . . . . 26

3 Equações Diferenciais Diferença Impulsivas 32

3.1 Introdução . . . . . . . . . . . . . . . . . . . . . . . . . . . . 32

3.2 Estudo de periodicidade . . . . . . . . . . . . 33

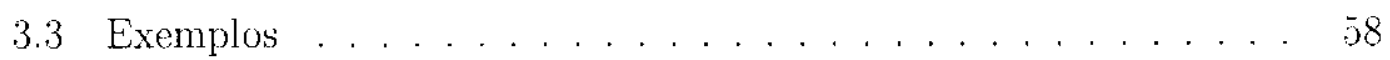

4 Soluções periódicas contra-fluxo $\quad 63$

$4.1 \mathrm{Um}$ caso linear . . . . . . . . . . . . . . . . 63

4.2 Estudo de um problema impulsivo . . . . . . . . . . 67

$\begin{array}{ll}\text { Referências Bibliográficas } & 79\end{array}$ 


\section{A Comissão Julgadora:}

Prof. Dr. Plácido Zoega Táboas

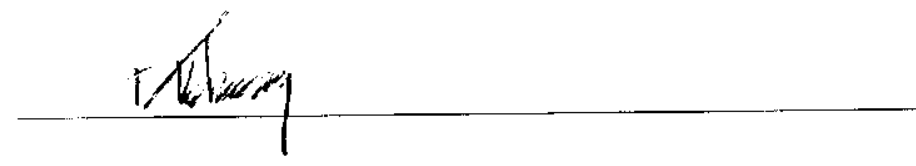

Prof. Dr. Luiz Augusto da Costa Ladeira

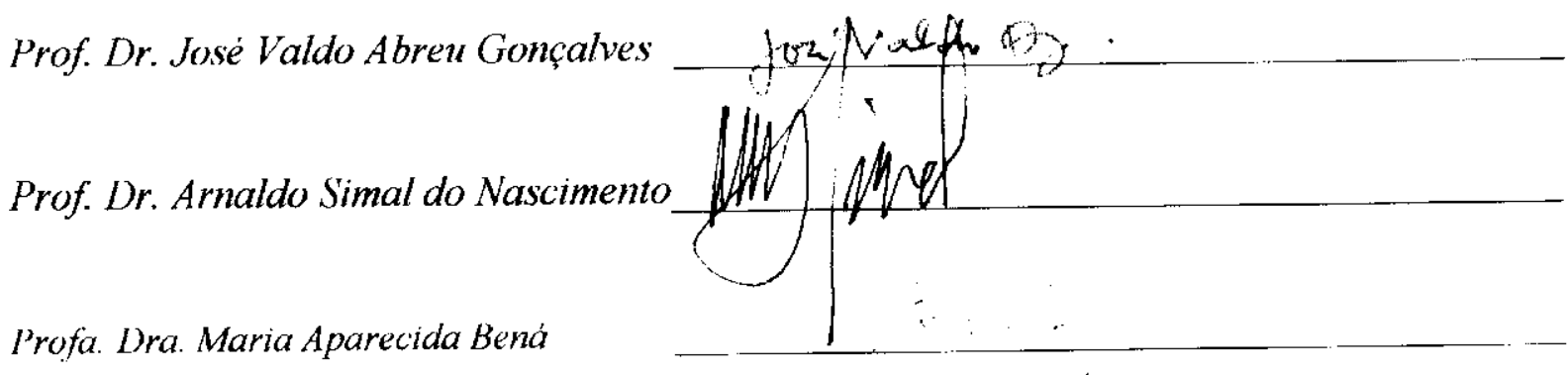


A minha querida mâzinha

"A coragem de latar nào vem do desejo de alcançar a vitória, mas do sentimento de poder compartilhar alegrias." 


\section{Agradecimentos}

Ao Prof. Dr. Pláciclo Zoega Táboas pela forma atenciosa e objetiva clue conduziu nossa orientação. Pelo incentivo, pela dedicarâo, paciência a amizade.

A Profa. Dra. Sandra M.S. de Godoy, pelo apoio, sinceridade c amizade.

Aos professores do Departamento de Matemática do ICMC-USP pelo apoio e pela inspiração que nos influenciaram nestes anos.

A todos os amigos, de graduação. de pós-graduação e tantos outros que me animaram e me ajudaram nesses anos.

As funcionárias da Secretaria de Pós-Graduaçõo e da Biblioteca pela simpatia, atençăo e eficieincia.

À minha família pelo carinho, pelo incentivo, pela paciência e pela credibilidade.

Ao Valdir, pela compreensão, pelo apoio e especialmente pelo amor que nos acompanhou nestes dias atarefados.

A FAPESP pelo apoio financeiro.

Eufim, a todos que direta ou indiretamente contribuíram para a realização deste trabalho. 


\begin{abstract}
We stablish conditions such that a certain non-linear impulsive differential cquation has an oscillatory solution. We also investigate the asymptotic belavior of such solutions. The existence of oscillatory solutions to a planar version of the above problem is acemphished.

By adding a self-supporting impulsive condition to a differential-difference equation in the plane, wo obtain sufficient conditions for the existence of periodic solutions.

A crucial role in the planar problems considered here is played by a known version of the negative feedback condition of the scalar problems. Such version suggests that all solutions in the future turn in the clock-wise sense aromed the origin. However we exhibit examples that show the existence of periodic: solutions whose orbits turn around the origin in the counter clock wise sense. Such solutions are called backset solutions.
\end{abstract}




\section{Resumo}

Estabeleccmos condiçōes para que uma cquação diferencial não linear impulsiva possua solução oscilatória. Estudamos o comportamento das soluçōes $x(l)$ deste problema culando $t \rightarrow \infty$. Alguns resultados sobre a existéneia de solução oscilatória para o caso de mma equação planar sào estabelecidos.

Acrescentando uma condição de impulso de anto-sustentação a nma equação diferencial diferença no plano obtemos condições suficientes para a existência de soluçào periódica.

Em papol crucial nos problemas planares considerados aqui é desempenhado por una versào já conbecida da condiçào de retro-alimentaçào negativa dos problemas escalares. Fsta versino sugere que todas as solugeos mo futuro giram no sentirlo horário ('m torno da origem. Exibinos, entretanto, exemplos que mostram a rexisteneia de soluçoes periódicas. cujas órbitas giran em torno da origem no sentido anti-horário, são as chamadas solıçoes contra-fluxo. 


\section{Introdução}

Lm sistema impulsivo é uma equação diferencial sujeita a uma condição adicional, que pode ser entendida como uma condição de contomo. Essa condiçăo a que provoca os impulsos, isto é, mudarcças bruscas de estado durante o desenvolvimento do processo definido pela equagão diferencial. Os impulsos são, na verdade, perturbações de tão curta duração que podem ser considerados instantâneos. Vários fenômenos naturais podem ser descritos pelas equações impulsivas, veja, por exemplo, [6!. [7] e [15].

Neste trabalho eonsideramos equaçoes com retardamento sujeitas a certas condiçônes de impulso. Essas condiçoes trazem algumas dificuldades que năo se apresentam no caso ordinário, já que os impulsos representam, em geral, descontinuidades de primciro tipo dos termos $x_{t}$ pertencentes ao espaco de fase. Assinn é necessário adaptar resultados clássicos da teoria das equaçóes diferenciais funcionais no espaço de fase $C\left([-r, 0]\right.$. s. $\left.^{n}\right)$. Também, no estudo da existéncia de soluçós periódicas pela análise de um operador de retorno os inpulsos perlem inviabilizar o uso direto dos princípios de ponto fixo mais conhecides.

Iniciamos este trabalho, com o Capítulo 1, destacando alguns fatos básicos da feria de sistemas impulsivos e listando os resultados que serão utilizados para desenvolver os capuítulos 2, 3 o 4. Definimos as equaçoes impulsivas o apresentamos dois tijes do impulso, acpueles em oue os instantes de impulso sào previamente 
estabelecidos e outros que dependem da solução atingir certos valores críticos.

No Capítulo 2 estudamos a existência e o comportamento assintótico de soluções oscilatórias de um problema impulsivo escalar com retardamento. Neste caso, os instantes de inpulso sào pré-fixados. É apresentado um estudo para um caso năo-lincar. No final do capítulo estabelecemos resultados iniciais que garantem a cxistencia de solução oscilatória para um caso planar.

Vo Capítulo 3, consideramos una equaşăo diferencial planar impulsiva com retardamento do tipo diferenga. A condição de inupulso é do tipo anto-sustentaço, segundo [15] descrita no Capítulo 1. Obtemos condiçöes para a existencia de soluçöes periódicas utilizando um teorema de ponto fixo. Apresentamos um exemplo e ainda estabelecemos condições para que as soluções periódicas dos problemas estudados scjam do tipo impulsivo.

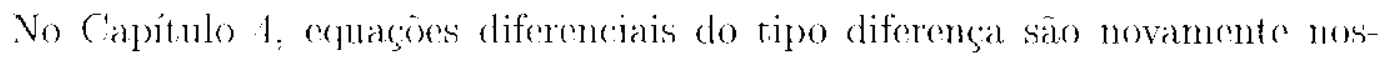
so objeto de estudo. Exibimos exemplos de equacôes satisfazendo as condiçoes estabelecidas no Capítulo 3, cujas órbitas podem apresentar a interessante propriedade de girar no sentido oposto ao sugerido pela hipótese (H1), contra-partida. planar da consagrada condição de retro-alimentaçâo negativa em equaçóes escalares. Denominamos estas soluçöes como soluçöes contra-fluxo. Veste capítulo exibinos um exemplo linear sem impulsos possuindo solucoes periódicas contrafluxo e definimos una condiçăo de inmpulso que garante a existencia de soluçóes periódicas contra-fluxo para uma equação diferencial diferença não-linesar. 


\section{Capítulo 1}

\section{Preliminares}

Este capítulo trata de listar e esclarecer alguns aspectos básicos sobre a teoria de sistemas impulsivos que scrâo necessários para a compreensão dos capitulos seguintes. Também traz resultados sobre as equaçoes difercnciais funcionais, já que nosso objetivo é estudar as equaçoes diferenciais impulsivas com retardanento.

Segundo [7], um sistema impulsivo, para o caso ordinário, pode ser representado da seguinte forma:

(i) Considera-se una equaçăo diferencial

$$
\dot{x}=f(t, x)
$$

onde $f: \mathbb{R}_{+} \times \Omega \rightarrow \mathbb{R}^{n}, \Omega \subset \mathbb{X}^{n}$ aberto, satisfaz alguma condição de unicidade para problemas de valores iniciais,

(ii) subronjuntos $M(t) \subset \Omega, l \in \mathbb{R}$. o

(iii) funçoes $A(l): M(l) \rightarrow \Omega, t \in \mathbb{R}_{+}$.

Lima solução desse problema passando pelo ponto $\left(t_{01}, x_{01}\right) \in \mathbb{R}_{1} \times \Omega$ coincide? com a solução $x(t)=x\left(t ; t_{0}, x_{0}\right)$ de $(1.1)$ por $\left(t_{0}, x_{0}\right)$ para $t \in\left(t_{0}, t_{1}\right]$, onde $l_{1}$ 
o menor valor de $l>t_{0} \operatorname{com} x\left(t_{1}\right) \in M\left(t_{1}\right)$. A funçäo $A\left(t_{1}\right)$ leva $x\left(t_{1}\right)$ em $x_{1}:=\Lambda\left(t_{1}\right) x\left(t_{1}\right)$. A partir deste instante. a solução coinciele com a solução $x(t)=$ $x\left(t ; t_{1}, x_{1}\right)$ de $(1.1)$ por $\left(t_{1}, x_{1}\right)$ para $t \in\left(t_{1}, t_{2}\right]$, onde $t_{2}$ é o menor valor de $t>$ $t_{1} \operatorname{com} x\left(t_{2}\right) \in M\left(t_{2}\right)$. A funçà $A\left(t_{2}\right)$ leva $x\left(t_{2}\right)$ em $x_{2}:=A\left(t_{2}\right) x\left(t_{2}\right)$ e assim sucessivamente.

O sistemi impulsivo descrito aqui pode ser denotado por

$$
\begin{gathered}
\dot{x}=f(t, x) \\
x(t) \in M(t) \Rightarrow x(t+)=A(t) x(t) .
\end{gathered}
$$

No caso em que $M(t)=M$ e $A(t)=A: M \rightarrow M$ não dependem de $t \in \mathbb{R}_{+}$ e a equação é autònoma, o problema impulsivo, segundo Myshkis, [10] e [15], é chantado de sistema impulsivo com condiça de auto-sustentação. Nos capítulos 3 o 4 vanos tratar especialmente deste tipo de impulso.

Podemos observar que uma solução de um sistema diferencial impulsivo pode ser:

a) uma funçăo contínua, se a curva integral $\left\{(t, x) t \geq t_{0}, x=x(t)\right\}$ não interceptar o conjunto $M(t)$ on se atingir nos pontos fixos do operalor $A(l)$ :

b) uma função contínua por partes tendo um número linito de descontinuidades de primeiro tipo se a curva encontrar $M(l)$ em am número finito de pontos cque não são pontos fixos do operador $A(t)$ :

c) uma funçào contínua por partes tendo um número contável de descontinuidades de primeiro lipo se a curva encontrar o conjunto $M(t)$ em un número contável de pontos que não sấo pontos fixos do operador $A(t)$.

Os instantes $t_{k}$ nos quais $I_{t}^{\prime}$ atinge $M(t)$ sia chanados momentos de im- 
pulso.

Em todo trabalho vamos utilizar a seguinte notação:

$$
\begin{aligned}
& Q_{1}:=\left\{\left(x_{1}, x_{2}\right): x_{1} \geq 0, x_{2} \geq 0\right\} \\
& Q_{2}:=\left\{\left(x_{1}, x_{2}\right): x_{1} \geq 0, x_{2} \leq 0\right\} \\
& Q_{3}:=\left\{\left(x_{1}, x_{2}\right): x_{1} \leq 0, x_{2} \leq 0\right\} \\
& Q_{4}:=\left\{\left(x_{1}, x_{2}\right): x_{1} \leq 0, x_{2} \geq 0\right\}
\end{aligned}
$$

Para estabelecermos resultados sobre a existência de solução periódica, se faz necessário alguns fatos e definições da teoria de equaçoes diferenciais funcionais e resultados sobre a existencia de ponto fixo que citaremos a seguir e que podem sor encontrados em [1].

Definição 1.0.1 Sejam X um espaço de Banach, U um subconjunto de $X$ ex um ponto de $\ell$. Dada uma aplicacäo $A: U \backslash\{x\} \rightarrow X$; o ponto $x \in U$ é dito um ponto ejetivo de $\mathbf{A}$ se existir una vizinhanca aberta $G \subseteq \mathrm{X}$ de $x$ tal que para todo $y \in G \cap U, y+x$, wiste um inteiro $m=m(y)$ tal que $A^{m} y \notin G \cap U$.

Teorema 1.0.1 Sejam $X$ um espaço de Banach, $K \subset X$ um comjunlo fechado, limitado, convero, de dimensão infinita, $A: K \backslash\left\{x_{0}\right\} \rightarrow K$ uma aplicaçäo completamente contína e $x_{0} \in K$ um ponto ejetivo de $A$, entäo existe um ponto fixo de $A$ em $\Lambda \backslash\left\{x_{0}\right\}$. 
Scja $C=C\left([-r, 0], \mathbb{R}^{2}\right)$ o espaço das funçòes contínuas definidas cm $[-r: 0]$, $r>0$, com imagem em $\mathbb{R}^{2}$ municlo da norma do sup. Consideremos as equaçós

$$
\dot{x}(t)=L x_{t}+f\left(x_{t}\right)
$$

$\theta$

$$
\dot{y}(t)-L y t
$$

onde $L: C \rightarrow \mathbb{R}^{2}$ ć uma aplicaçào linear contíntua, $\int: C \rightarrow \mathbb{R}^{2}$ ć completamente contínua, continuamente diferenciável. $f(0)=0, f^{\prime}(0)=0$. Existe uma matriz $2 \times 2, \eta(\theta), \theta \in[-r, 0]$, cujos elementos são de variação limitada e satisfaz as condiçòes: $\eta(0)=0, \eta$ é contínua à esquerda em $(-r ; 0)$, tal que

$$
L(\phi)-\int_{-r}^{0} d \eta(\theta) \phi(\theta), \quad \phi \in C
$$

Denotemos por $x_{t}(. ; \phi)$ c $y_{l}(. ; \phi)$ as soluçös de (1.2) e (1.3) respectivamento, com a condiçào inicial $\phi \in C$.

Seja $T(t), t \geq 0$ a apticaçào solução de (1.3), isto é:

$$
T(t) \phi=y_{t}(\therefore \phi), \quad \phi \in C
$$

Para qualquer autovalor $\lambda$ de (1.3), ou scja, $\lambda$ satisfaz

$$
\operatorname{det} \Delta(\lambda)=0, \quad \Delta(\lambda)=\lambda I-\int_{r}^{0} e^{\lambda \theta} d \eta(\theta)
$$

o espaco $C$ pode ser decomposto por

$$
C=P_{\lambda} \odot Q_{\lambda}
$$

onde $P_{\lambda} \cdot Q_{\lambda}$ são invariantes pelo operador $T(t), t \geq 0$. O anto-espace $P_{\lambda}$ tem dimensão tinita, veja capítulo 7 de $\mathrm{Aj}_{\mathrm{j}}$. 
Se $A$ é o gerador infinitesimal de $T(t)$, entàa $P_{\lambda}=N(\lambda I-A)$ e $Q_{\lambda}=I(\lambda I-A)$. Seja $\Pi_{\lambda}$ a projeção de $C$ em $P_{\lambda}$. Se $u$ é uma solução náa trivial de $\Delta(\lambda) u=0$ e o vetor $v$ co man soluçäo năo trivial de $v \Delta(\lambda)=0$, ontão as funçoes $\rho(l)=e^{\lambda \ell} u, l \in$ $[-r, 0] \odot \sigma(s)=v e^{-\lambda s}: s \in[0, r] \operatorname{geram} N(\lambda I \cdots A) \propto N\left(\lambda I-A^{*}\right)$, respectivamente, onde $A^{*}$ é o adjunto de $A$. Para $\phi \in C . \dot{O}-\phi^{P_{\lambda}}-\phi^{Q_{\lambda}}$, a componente $\phi^{P^{2}}$ é dada por

$$
\mathrm{I}_{\lambda} \phi=\phi^{P_{\lambda}}=\langle\sigma, \phi\rangle_{\rho}
$$

onde $\langle.$.$\rangle denotia a forma bilinear definida por: se C^{*}=C\left([0, r], \mathbb{R}^{2 *}\right)$, onde $R^{2 *}$ é o plano dos vetores linhas, para $t \in C^{\times}$e $\phi \in C$

$$
\langle\psi, \phi\rangle=\psi(0), \phi(0)-\int_{-r}^{0} \int_{--r}^{\theta} \psi(\theta-\varepsilon)[d \eta(\varepsilon)] \phi(\theta) d \theta
$$

lembrando que ". representa o produto escalar de $\mathbb{R}^{2}$.

Como os impulsos representam , em geral, descontinuidades de primciro tipo dos termos $x_{t}$, precisaremos de um espaço mais geral que $C\left([a, b], \mathbb{R}^{2}\right)$. Nos problemas propostos no Capítulo 4, por exemplo, consideraremos o espaço de fases definido por $G=G\left([a, b], \mathbb{R}^{2}\right)$ que é o espaço das funçós regradas. Lina introdusão sobre as propriedades desse espaço pode ser encontrada em [5]. Alguns fatos básicos apresentaremos aqui.

Definição 1.0.2 Seja $X$ um espaço de Banach e $f:[a, b] \rightarrow X$. Dizenos que $f$ é uma funçāo regrada, $f \in G([a, b], X)$, se tem somente descontinuidades de primeiro tipo, isto é, para todo $t \in[a, b)$ existe $f(t+)$ e para todo $t \subset(a, b]$, existe $f(t-)$. 
Proposição 1.0.1 $f:[a, b] \rightarrow X$ é uma funçăo regrada se, e somente se, $f$ é o limite uniforme de funçòs escadas.

Lema 1.0.1 Se $\int \in G([a, b], X)$ entäo o conjunto de descontinuidades de $f$ enumeráuel e $C([a, b], X) \subset G([a, b], X)$.

Como o limite uniforme de funçoes regradas é uma função regradit, considerando $C r([a, b], X)$ com a norma

$$
|f|=\sup _{a \leq t \leq b}|f(l)|
$$

temos que este espaço é de Banach. 


\section{Capítulo 2}

\section{Estudo sobre Soluções}

\section{Oscilatórias}

\subsection{Introdução}

Nosso objetivo, neste capítulo, é apresentar resultados sobre soluções oscilatórias e propriedades assintóticas de soluções de uma equação diferencial impulsiva. com retardamento.

Inicialmente, vamos considerar o problema sem impulso. Em [8] o [9], por exemplo, são estabelecidos resultados sobre oscilação e atratividade global para uma equação escalar não linear da forma

$$
x^{\prime}(t)=-p(t) f(x(t-\tau))
$$

onde $\tau$ é una constiante positiva e $p:[0, \infty) \rightarrow[0, \infty)$ e $f: \mathbb{R} \rightarrow \mathbb{R}$ são funçös contínuas com $x f(x)>0$ se $x \neq 0$.

Em [3] e [14], resultados sobre oscilação e comportanmento assintótico são estabelecidos quando uma condição do impulso é acrescentada à equação diferencial com retardamento: 


$$
\dot{x}(t)=-\sum_{i=1}^{n} p_{i}(t) x\left(t-\tau_{i}\right), \quad l \neq t_{k}
$$

a condição de impulso ć dada por

$$
\Delta x\left(t_{k}\right)=x\left(t_{k}+\right)-x\left(t_{k}\right)=b_{k} x\left(t_{k}\right), \quad k=1,2, \cdots,
$$

onde $p_{i}(t) \in C([0, \infty) ; \mathbb{R}), i=1,2, \ldots, n, 0 \leq \tau_{1}<\tau_{2}<\ldots<\tau_{n}, b_{k}$ são constantess, $k=1,2, \ldots, \phi$ é uma dada função inicial e os instantes de impulso satisfazem:

$$
0<t_{1}<t_{2}<\cdots<t_{k}<\cdots, \quad \lim _{k \rightarrow \infty} t_{k}=\infty
$$

Na mesma linha de investigacão dos trabalhos citados acima, vamos apresentar, na seção 2.2, un estudo sobre soluçoes oscilatórias para a equação diferencial não-linear (2.1) sujeita à condição de impulso (2.3). Queremos encontrar condições suficientes para que soluções oscilatórias e não-oscilatórias tendam a zero quando $t \rightarrow \infty$.

Para isto se torna nocessário introduzir algumas definiçoes que estão descritas cm [14].

Sejam $\sigma \geq 0$ oP $C_{\sigma}$ o conjunto das funções $\phi:\left[\sigma-\tau_{n}, \sigma\right] \rightarrow \mathbb{R}$ que são contínuas no conjunto $\left[\sigma-\tau_{n}, \sigma\right] \backslash\left\{t_{k} . k=1,2, \cdots\right\}$, podendo ter descontinuidades de primeiro tipo nos pontos $t_{k}$ situados no intervalo $\left(\sigma-\tau_{n}, \sigma\right]$ e sendo contínuas à esquérda.

Definição 2.1.1 Para $\sigma \geq 0$ e $\theta \in P C_{\sigma}$, uma funçào $x$ é soluçăo de (2.2) $e$ (2.3) satisfazendo a condicäo de valor inicial

$$
x(l)=\phi(t), \quad t \in\left[\sigma-\tau_{n}, \sigma_{j}^{i}\right.
$$

no intervalo $: \sigma, a)$, se $x:\left[\sigma-\tau_{n}, a\right) \rightarrow \mathbb{R}$ satisfaz (2.4)e 
A) para $l \in(\sigma, a), \quad t \neq t_{k}, t \neq t_{k}+\tau_{i}, x(t)$ é continuamente diferenciáuel e salisfaz (思):

B) para $t_{k} \in[\sigma, a), x\left(t_{k}^{-1}\right), x\left(t_{k}^{-}\right)$cxistem ex $x\left(t_{k}^{-}\right)=x\left(t_{k}\right)$ e satisfaz (2.3);

C) para $t \in(\sigma, a), t \neq t_{k}, t=t_{k}-\tau_{i}, x(t)$ é contínua e $x^{\prime}\left(t^{+}\right), x^{\prime}\left(t^{-}\right)$ existem.

A existència c a micidade de solução para o problema (2.2), (2.3) e (2.4) são garantidas pelo Lema 1 de [14].

Definição 2.1.2 Uma solução de (2. (2) e (2.3) é chamada não oscilatória se é positiva no futuro ou nexgativa no futuro. Caso contrário é chamada oscilatória.

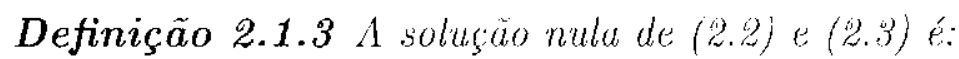

a) estável, se para qualquer $\Xi>0, \sigma \geq 0$, existe $\delta=\delta(\varepsilon, \sigma)>0$ tal que $\phi \in P C_{\sigma} e \mid \phi \|<\delta$ implica $\mid x(t):<z$ para $\iota \geq \sigma$, onde $x(t)-x(t, \sigma, \phi)$ é a

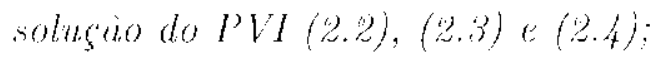

b) assintoticamente estável se for estável e para qualquer soluçäo de (2.9) e (2.3), temos $\lim _{t \rightarrow x} x(t)=0$.

Na seção 2.3, consideramos um problema planar correspondente ao escalar, cstudado na seção 2.2 , dado por

$$
\begin{gathered}
x(l)-p(t) F(x(t-\tau)), \quad t \neq t_{k}, \\
\Delta\left(x\left(t_{k}\right)\right)=b_{k} x\left(t_{k}\right), \quad k=1,2, \cdots,
\end{gathered}
$$


onde $x-\left(x_{1}, x_{2}\right) \in \mathbb{R}^{2}, F^{\prime}=\left(F_{1}^{\prime}, F_{2}\right)$ c uma funçà de classe $C^{2}$ de $\mathbb{R}^{2}$ em $\mathbb{R}^{2}$, $\tau>0$, constante, $p:[0, \infty) \rightarrow[0, \infty)$ contínua, $b_{k}$ constantes, $k=1,2, \cdots$, $0<t_{1}<t_{2}<\cdots<t_{k}<\cdots$, salisfazendo $\lim _{k, \infty} t_{k}=\infty$.

Alguns resultados solbre soluçoes oscilatórias são estabelecidos.

\subsection{Um problema impulsivo escalar e não-linear}

Em [9] o comportamento de soluções da equação diferencial não-linear e nãoantônoma com retardamento

$$
x^{\prime}(t)=-p(t) f(x(t-\tau))
$$

onde $\tau>0, p:[0, \infty) \rightarrow[0, \infty)$ contínua, $f: \mathbb{R} \rightarrow \mathbb{R}$ contínua, com a propriedade

$$
x f(x)>0, \text { se } x \neq 0
$$

é estudado. Vamos inicialmente estabelecer um resultado para a equação (2.5), (1ue goneraliza o Lema 2.1 de [9]. Vamos tomar as soluções de (2.5) com a condição inicial dada por

$$
x(t)=\phi(t) . \quad-\tau \leq t \leq 0
$$

onde $\phi$ é uma função contímua. Neste caso é fácil ver que existe solução de (2.5) definida no futuro.

Lema 2.2.1 Se

$$
\int_{0}^{\infty} p(s) d s=\infty
$$

então, toda soluráno nào-oscilatória de (2.5) tende a zero quando $t \rightarrow \infty$.

Demonstração: Seja $x(t)$ uma solução nãu-oscilatória de (2.5). Então existe um instante $t^{*}$ tal que $x(t)$ tem sinal constante para qualquer $t \geq t^{*}-t$. Vamos considerar $x(t)>0$ para $t \geq t^{\times}-\tau$ : o caso em que $x(t)<0$ ć análogo. Assim, 
$x^{\prime}(t) \leq 0$ para todo $t \geq t^{*}$, o que implica $x(t)$ ser náo-crescente em $\left[t^{*}, \infty\right) \mathrm{e}$ portianto, dewe existir $\alpha \geq 0$ tal que $\lim _{\ell \rightarrow \infty} x(b)=\alpha$. Mostremos que $\alpha=0$.

De fato, se $\alpha>0$, coltio para (qualduer $l>t^{*}+\tau, x(t) \geq \alpha$. Soja $\beta=x\left(t^{*}\right) \geq$

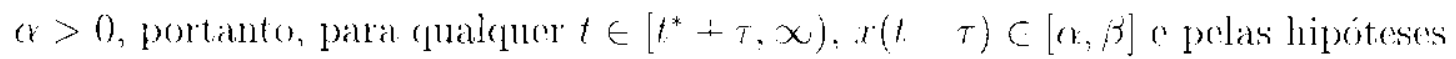
sobre a $f$, deve existir $\sigma>0$, lal (que $\sigma=\min _{\left.x \varepsilon_{i} e, \beta\right]} f(x)>0$.

Logo, para $t \geq t^{*}+\tau, l^{\prime}(b) \leq-p(t) \sigma$. Integrando essa desigualdade de $l^{*}+\tau$ a t, obtenros

$$
x(t)-x\left(t^{*}: \tau\right) \leq \sigma \int_{l * 1 \tau}^{t} p(s) d s
$$

Fazendo $t \rightarrow \infty$ na desigualdade acima. cheganos a uma contradição, já que o lado escuerdo da desigualdade tonde para um limite finito e o lado direito para $\cdots \infty$.

() Lema 2.2.1 é mais geral que o Lema 2.1 de [9], pois nào exigimos aqui que $f$ seja năo-decrescente e nem que $: f(x)|<| x \mid$ se $x \neq 0$. Para contuir que as soluçoes de (2.5) trudem a zero quando $t \rightarrow \infty$, citaremos o teorema abaixo que pode ser encontrado nm [9].

Teorema 2.2.1 Se fé uma dada funçào contínua näo-decrescente que satisfaz

$$
|f(x)|<|x| \text { se } x \neq 0 \text {. }
$$

p satisfaz (2.6) e, para l suficientemente grande satisfaz

$$
\int_{l-\tau}^{t} p(s) d s \leq \frac{3}{2}
$$

Então, toda soluçăo de (息.5) tende a zero quando $t \rightarrow \infty$.

Podemos afirmal que em vista das suposicöos do resultado arima a solução 
nula de (2.j) é globalmente assintoticamente estável.

Consideremos agora o problema dado por

$$
x^{\prime}(t)=-p(t) f(x(t-\tau)) . \quad l \neq t_{k},
$$

com a condiçãor de impulsos

$$
\Delta x\left(t_{k}\right)=x\left(t_{k}^{+}\right)-x\left(l_{k}\right)=b_{k} x\left(t_{k}\right), \quad k=1,2, \cdots
$$

onde $\tau>0, b_{k}, k=1,2, \ldots$, são constantes, os instantes de impulso são pré-fixados e satisfazen $0<t_{1}<t_{2}<\cdots<t_{k}<\cdots$ o $\lim _{k \rightarrow \infty} t_{k}=\infty$. As hipóteses sobre a $f$ e $p$ säo as mesmas cestabelecidas para (2.5).

Seja

$P C=\{\phi:[-\tau, 0] \rightarrow \mathbb{R}, \phi$ tem um número finito de descontinuidades que são de primeiro tipo, $\phi$ é contínua à esquerda em cada ponto de clescontinuidade

A definição de solugão e de solução oscilatória para este problema são similaress às definições mencionadas nà seção 2.1.

Observação 2.2.1 É evidente que para certos problemas do tipo (9.9) e (2.10) existem soluçöes não-oscilatórias. Por exemplo, se considerarmos $p(t) \equiv 1, t_{k}=$ $k . b_{k}=1, k=1,2, \cdots$ ef limitada, digamos $\mid f(x) \leq l$, para todo $x \in \mathbb{R}, l>0$, e a condiçào inicial $\phi \equiv 2 l$. É fácil ver que este problema dá origem a uma soluçâo positiva e, portanto, näo-oscilatória.

Lema 2.2.2 Suponhamos que

$$
t_{k}-t_{k-1}>\tau>0, \quad k=1,2,3, \cdots
$$




$$
|f(x)| \geq \lambda|x| . \quad \text { para algurn } \lambda>0 .
$$

Se

$$
\limsup _{i \rightarrow \infty} \frac{\lambda}{1+b_{i}} \int_{t_{2}}^{t_{i}+\tau} p(s) d s>1 .
$$

Entâo, toda soluçâa dé(2.9) e (2.10) é oscilatória.

Demonstração: Suponhamos, por absurdo, que existia uma solução $x(t)$ de (2.9) Q (2.10) 1lăo-oscilatória. Sem perda de gemeralidade, vanos supor que $x(t)>0$, para todo $t \geq t^{*}$, para un certo $t^{*}$. Assim. $x^{\prime}(t) \leq 0$ para $t \neq t_{k}, t \geq t^{*}+\tau$. Integrando $(2.9)$ de $t_{i}$ a $t_{i}+\tau . t_{i} \geq t_{l}$, onde $t_{l}=\min \left\{t_{k} ; t_{k}>t^{*}+2 \tau\right\}$ temos

$$
\begin{aligned}
& 0=x\left(t_{i}+\tau\right)-x\left(t_{i}^{+}\right)+\int_{t_{i}}^{t_{i}+\tau} p(s) f(x(s-\tau)) d s \\
& \geq x\left(l_{i}+\tau\right)-x\left(l_{i}^{+}\right)+\lambda \int_{t_{i}}^{t_{2}+\tau} p(s) x(s-\tau) d s \\
& \quad \geq x\left(t_{i}+\tau\right)-x\left(t_{i}^{+}\right)+\lambda x\left(t_{i}\right) \int_{l_{i}}^{t_{i}+\tau} p(s) d s
\end{aligned}
$$

a última desigualdade segue do fato de $x(t)$ ser näo-crescente nos intervalos da forma $\left(l_{t}, l_{i+1}\right)$, com $i=l, l+1, \cdots$. Etilizando $(2.10)$, temos

$$
x\left(t_{i}+\tau\right)+x\left(t_{i}^{+}\right)\left[\frac{\lambda}{1+b_{i}} \int_{l_{i}}^{t_{i}+t} p(s) d s-1\right] \leq 0
$$

isso é uma contradição, já que estamos assumindo $x(t)>0$ e (2.12).

Agora queremos estabelecer um resultado como o Teorema 2.2.1 para o caso impulsivo. Para isto seräo necessários alguns lemas.

Lema 2.2.3 Suponhamos que

$$
\sum_{k-1}^{\infty}\left|b_{k}\right|<\infty
$$


e que existem constantes positivas $A, Q_{1}, Q_{2}$ er $r \in[0, \tau]$ tais que

$$
|f(x)| \leq A x \mid: \quad A\left(Q_{1}+Q_{2}\right)<1
$$

para t suficientemente estao salisfeitus as segumtes condiçörs

$$
\int_{l}^{t+\tau} p(s) d s \leq Q_{1}, \quad \int_{l \tau}^{t-r} p(s+\tau) d s \leq Q_{2}, \quad p(t+\tau) \neq 0 .
$$

Entäo, toda soluçäo oscilatória de (名9) e (2.10) tende a zero quando $t \rightarrow \infty$.

Demonstraçāo: Soja $x(t)$ uma soluçào oscilatória de (2.9) e (2.10) e seja

$$
\nu=\limsup _{t \rightarrow \infty}|x(t)|
$$

Primeiramente vamos provar que $\nu<\infty$. Para isto, suponhamos, por contradição, quo $1=\infty$.

Seja $N$ un inteiro positivo, suficientemente grande tal que

$$
\sum_{k=N}^{\infty}\left|b_{k}\right|<\frac{1-A\left(Q_{1}+Q_{2}\right)}{2}
$$

o que as hipóteses (2.15) sobre a função $p(t)$ estejam satisfeitas para todo $1 \geq$ $t_{N}+\tau$ c que lambém valha

$$
\sup _{t_{N}+\tau \leq s \leq t}|x(s)|=\sup _{t_{N} \leq s \leq t}|x(s)|, \quad t \geq t_{N}+\tau .
$$

Observamos que se essa igualdade não ocorresse para nenhum $N$, entäo para dado $1>t_{N}$ teríanos

$$
\sup _{t_{N} \leq s \leq t}|x(s)|=\sup _{t_{N} \leq s \leq t_{N} \cdot \tau}|x(s)|, \quad \forall N
$$

c isso contradiz o latto de $\nu=\infty$.

Agora. vamos definir a seguinte funça

$$
y(t)=x(t)-\int_{l-\tau}^{t \cdots r} p(s-\tau) f(x(s)) d s-\sum_{t_{N} \leq t_{k}<l} b_{k} x\left(t_{k}\right) .
$$


Então, para $t \neq t_{k}, \quad t \neq t_{k}+\tau, \quad k=1,2, \cdots$, temos

$$
y^{\prime}(t)=-p(t-r+\tau) f(x(t-r))
$$

cassim, para $t \geq t_{N}+\tau$. usando as hipóteses (2.14) e (2.15), 1emos

$$
\begin{array}{r}
|y(t) ! \geq| x(t)\left|-4 \int_{l-\tau}^{l-r} p(s+\tau)\right| x(s)\left|d s-\sum_{t_{N} \leq t_{k}<t}\right| b_{k} x\left(l_{k}\right) \\
\geq|x(l)|-4 \sup _{t-r \leq s<t}|x(s)| \int_{l-t}^{t \cdots r} p(s+\tau) d s-\sup _{t_{k} \leq s \leq t}|x(s)| \sum_{k=N}^{\infty}\left|b_{k}\right| \\
\geq|x(t)|-4 \sup _{t_{N} \leq s \leq t}\left|x(s)_{l} Q_{2}-\sup _{l_{N} \leq s \leq t}\right| x(s)\left|\sum_{k=N}^{\infty}\right| b_{k} \mid \\
=|x(t)|-\left(A Q_{2}+\sum_{k=N}^{\infty}\left|b_{k}\right|\right) \sup _{l_{N} \leq s \leq l}|x(s)| .
\end{array}
$$

$\log 0$

$$
\sup _{t_{N}}|y<s<l| y(s)\left|\geq\left(1-A Q_{2}-\sum_{k=N}^{\infty}\left|b_{k}\right|\right) \operatorname{sip}_{l_{N} \leq s \leq l}\right| x(s) \mid .
$$

Portanto, limsup $; x|y(t)|=\infty$.

Como $x(t)$ ć oscilatória e para $k \geq N$ vale

$$
x\left(t_{k}^{+}\right) x\left(t_{k}\right)=x^{2}\left(t_{k}\right)\left[1+b_{k}\right] \geq 0
$$

já que $b_{k} \rightarrow 0$ quando $k \rightarrow \infty$, ontäo $x(l)$ deve ter zeros arbitrariamente grandes. De $(2.18)$ e da hipótrese $p(t+\tau) \neq 0$ para $t \geq t_{N}+\tau$ temos que $y^{\prime}(t)$ também oscila e possui zeros arbitrariamente grandes. Logo. existe $\xi>t_{N}+2 \tau$ tal que

$$
|y(\xi)|=\sup _{t_{j}-\tau \leq s \leq \xi}|y(s)| \quad 0 \quad y^{\prime}(\xi)=0=-p(\xi-r+\tau) f(x(\xi-r)) .
$$

Por propriedade da função $f$, temos $x(\xi-r)=0$. Integrando $(2.18)$ de $\xi-r$ a $\xi$ segue? qur 


$$
y(\xi)-y(\xi-r)=-\int_{\xi-r}^{\xi} p(s-r+\tau) f(x(s-r)) d s,
$$

usando a definição (2.17) de $y$, temos

$$
\begin{aligned}
y(\xi)=x(\xi-r)-\int_{\xi-r-\tau}^{\xi-2_{r}} p(s+\tau) f(x(s)) d s & \\
& \quad-\sum_{t_{N} \leq t_{k}<\xi-r} b_{k} x\left(t_{k}\right)-\int_{\xi-2 r}^{\xi-r} p(s+\tau) f(x(s)) d s,
\end{aligned}
$$

01 seja,

$$
y(\xi)=-\int_{\xi-r-\tau}^{\xi-r} p(s+\tau) f(x(s)) d s-\sum_{t_{N} \leq t_{k}<\xi \cdots r} b_{k} x\left(t_{k}\right) .
$$

Por (2.14) o (2.15), podemos verificar que

$$
|y(\xi)| \leq A \int_{\xi-r-\gamma}^{\xi-r} p(s+\tau)|x(s)| d s+\sum_{t_{N} \leq t_{k}<\xi-r}\left|b_{k} x\left(t_{k}\right)\right|
$$

donde

$$
|y(\xi)| \leq\left(A Q_{1}+\sum_{k-N}^{x}\left|b_{k}\right|\right) \sup _{t_{N} \leq s \leq \xi}|x(s)| .
$$

De (2.19) e (2.20) segue que

$$
\left(1-.1\left(Q_{1}+Q_{2}\right)-2 \sum_{k=N}^{\infty}\left|b_{k}\right|\right) \sup _{i, \leq s \leq \xi}|x(s)| \leq 0
$$

o que contradiz (2.16) e portanto $l<\infty$.

Mostremos agora que $\nu=0$. Para isto vamos definir a funçâo

$$
z(t)=x(t)+\int_{l-r}^{l-\tau} p(s+\tau) f(x(s)) d s+\sum_{l_{k} \geq t} b_{k} x\left(t_{k}\right) .
$$


É fácil ver que $z(t)$ é uma aplicaçào limitada, já que $v<\infty$. Para $t$ suticientemente granda,

$$
|z(t)| \geq|x(t)|-A Q_{2} \sup _{t-\tau \leq s \leq t}|x(s)|-\left|\sum_{t_{k} \geq t} b_{k} x\left(l_{k}\right)\right|
$$

donde

$$
\rho=\limsup _{t \rightarrow \infty}|z(t)| \geq\left(1-A Q_{2}\right) \nu
$$

Por outro lado, como $z^{\prime}(t)=-p(t-r+\tau) f(x(l-r))$, para $l \neq l_{k} \mathrm{e} l \neq$ $t_{k}+\tau$, temos que $z^{\prime}(t)$ oscila e tem zeros arbitrariamente grandes, logo existe uma sequência $\left(\xi_{m}\right)$ lal que

$$
\lim _{n \rightarrow \infty} \xi_{m}=\infty, \quad \lim _{m \rightarrow \infty}\left|z\left(\xi_{m}\right)\right|=\rho: \quad z^{\prime}\left(\xi_{m}\right)=0, \quad m=1,2,3, \cdots
$$

Integrando $z^{\prime}(t)$ de $\xi_{m}-r$ a $\xi_{m}$, usando a definição (2.21) e como $x\left(\xi_{m}-r\right)=0$, obtenios

$$
z\left(\xi_{m}\right)=-\int_{\xi_{m-r-\tau}}^{\xi_{m-r}-r} p(s+\tau) f(x(s)) d s+\sum_{t_{k} \geq \xi_{m}-r} b_{k} x\left(t_{k}\right)
$$

portinto

$$
\left|z\left(\xi_{m}\right)\right| \leq A Q_{1} \sup _{\xi_{m}-2 \tau \leq s \leq \xi_{m}}|x(s)|+\left|\sum_{t_{k} \geq \xi_{m}-r} b_{k} x\left(t_{k}\right)\right|
$$

o que implica

$$
\rho \leq A Q_{1} \nu
$$

Somando as desigualdades (2.22) e (2.23), obtemos

$$
\left[1-A\left(Q_{1}-Q_{2}\right)\right] \nu \leq 0
$$

Segue de (2.14) que $y=0$ o que conclui a provd.

En vista dos lemas 2.2 .2 e 2.2.3, podemos estabelecer o seguinte teorema. 
Teorema 2.2.2 Se (2.11) e (2.13) estiverem satisfeitas e se existirem constantes positivas, $A, Q_{1}, Q_{2}, \lambda$ er $\left.C^{\ulcorner} 0, \tau\right]$, tais que

$$
A\left(Q_{1}+Q_{2}\right)<1, \quad \lambda|x| \leq|f(x)| \leq A|x|
$$

satisfazendo (2.12) e (9.15), endüo loda soluçăo de (2.9) e (2.10) tende a zero quando $t \rightarrow \infty$.

Cono mencionamos na introducajo, o resultado obtido acima ó mma extensäo à equaçäo nào lincar (2.9) de resultados de [3], [13] e [14], que so restringem a equaçoes lineares. Veste caso o problema (2.9) e (2.10) apresenta somente soluções oscilatórias. Veremos a scguir que podemos obter a mesma conclusão deste teorema impondo algumas hipóteses diferentes na função $f$, na função $p$ e nos impulsos quando nos concentramos também no estudo das soluçòes nãooscilatórias de (2.9) (2.10).

Lema 2.2.4 Suponhamos que

$$
b_{k}^{\dagger} \rightarrow 0
$$

onde $b_{k}^{+}-\max \left\{b_{k}, 0\right\}$,

$$
t_{k}-t_{k-1} \geq \tau>0
$$

para $k$ suficientemente grande $e$

$$
\mu(t) \geq c ;
$$

para $t$ suficientemente grande, onde c uma constante positiva. Seja $f$ nüodecrescente, satisfazendo

$$
|f(x)|>\lambda \mid x, \quad x \neq 0, \quad \lambda>0 \quad \text { constante. }
$$

Entáo, loda solugăo nào-oscilatória de (20.9) (D.10) tende a zero quando $l \rightarrow \infty$. 
Demonstração: Soja $x(t)$ uma solução não-oscilatória de (2.9) e (2.10). Então cxiste un $t^{*} \geq \tau$ tal ane $x(t)$ tem sinal constante para todo $t \geq t^{*}-\tau$. Vamos supor. sem perda de generalidade que $x(l)>0, \forall t \geq t^{*}-\tau$. Isso implica que

$$
x^{\prime}(t)=-p(t) f(x(t-\tau))<0 . \quad \forall t>t^{*}, \quad t \neq t_{k}
$$

Mostremos que, para $k$ suficientemente grande, $x\left(t_{k}^{+}\right) \geq x\left(t_{k+1}^{+}\right)$, ou seja, além da solução $x(t)$ ser decrescente nos intervalos abertos $\left(t_{k}, t_{k+1}\right)$, para $k$ suficientemente grande, também a seqüência $\left(x\left(t_{k}^{1}\right)\right)$ será não crescente para $k$ suficiontemente grande. Basta pensarmos em $b_{k}>0$. De fato, usando (2.10) temos que

$$
x\left(l_{k}\right)=\frac{1}{b_{k}+1} \cdot\left(t_{k}\right)
$$

e para $k$ suficientemente grande, vale

$$
x\left(t_{k}\right)>\frac{1}{b_{k}+1} x\left(t_{k+1}\right)
$$

É claro que $x\left(t_{k+1}\right)>\frac{1}{b_{k}+1} x\left(t_{k+1}\right)$ para $k=1,2.3 \cdots$. Portanto

$$
x(t)>\frac{1}{b_{k}+1} x\left(t_{k+1}\right), \quad \text { para } t \in\left[t_{k}-\tau, t_{k \cdot 1}\right]
$$

Vanos agora comparar o quanto a funçào $x(t)$ decresce cm $\left(t_{k}, t_{k+1}\right]$ em relação ao quanto ela salta em $t_{k+1}$. Usando (2.25), (2.26),(2.27) e a desigualdade acima, para $k$ suficientcemente grande temos

$$
\left|x^{\prime}(l)\right|>c \lambda|x(t-\tau)| \geq c \lambda \frac{1}{b_{k}+1} x\left(t_{k+1}\right), \quad t \in\left(t_{k}, t_{k-1}\right),
$$

portanto

$$
\left|x^{\prime}(t)\right|\left(t_{k 11}-t_{k}\right)>c \lambda \frac{1}{b_{k}+1} x\left(t_{k, 1}\right) \tau \geq b_{k+1} x\left(t_{k+1}\right)=\Delta x\left(t_{k+1}\right)
$$


o que conclui a prova da desigualdade $x\left(t_{k}^{+}\right) \geq x\left(l_{k+1}^{+}\right)$, e implica que $x(t)$ é limitada, isto $c$ existe $M>0$, lal $($ gue $: c(t) \leq M$ para todo $t \geq 0$.

Mostirenos que existe lime ra $x(1)$. Para isto vamos provar que

$$
\limsup _{t \rightarrow \infty} x(t)=\liminf _{t \rightarrow \infty} x(t)
$$

De fato, como a scquência $x\left(t_{k}^{-}\right)$é decrescente e limitada para $k$ suficientemente grande, então existe $\alpha<\infty$ tal que $\lim _{k-1 \times x} x\left(t_{k}^{+}\right)=\alpha$. Pelo comportamento da solução, temos quo $\limsup _{t \rightarrow \infty} x(t)=\lim _{t \rightarrow \infty} x\left(t_{k}^{+}\right)$.

Soja $\beta=\liminf _{t \rightarrow \infty} x(l) \leq \alpha$. Suponhamos, por absurdo, que $0 \leq \beta<\alpha$. Tomando $\varepsilon=\frac{\alpha-\beta}{3}>0$, existe uma sequencia $\left(\lambda_{j}\right)$ crescente, tal que $\lambda_{j} \rightarrow \infty$ quando $j \rightarrow \infty$ e existe $K_{1}$ satisfazendo $x\left(\lambda_{j}\right) \in V_{s}(\beta)$, para todo $j \geq K_{1}$, onde $V_{\varepsilon}(\beta)$ ć o intervalo aberto $(\beta-\varepsilon, \beta+\varepsilon)$.

Vamos construir a subsequência $\left(t_{k_{j}}\right)$. tomando $t_{k_{j}}$ como o primeiro instante de impulso tial que $t_{k_{j}} \geq \lambda_{j}$. Assim

$$
l_{k_{1}} \leq l_{k_{2}} \leq \cdots \leq l_{k_{i}} \leq \cdots, \quad \lim _{j \rightarrow \infty} l_{k_{i}}=\infty
$$

Portante $x\left(t_{k_{j}}\right)<x\left(\lambda_{j}\right)<\beta+\varepsilon<\alpha-\varepsilon$. Decorre de (2.24) que

$$
x\left(t_{k_{j}}^{+}\right)=\left[1+b_{k_{j}}\right] x\left(t_{k_{j}}\right)<\alpha-\varepsilon ;
$$

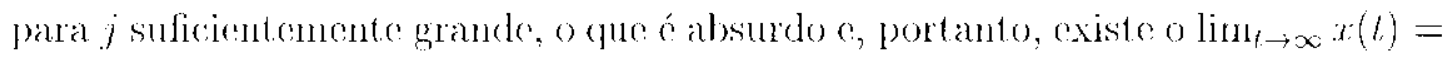
$\alpha$.

Mostremos que $\alpha=0$. Como $x(t)$ é limitada e vale (2.24), segue que $\Delta x\left(t_{k}\right) \rightarrow$ 0 , quando $k \rightarrow \infty$. Suponhamos, novamente por absurdo, que $\alpha>0$, então para $k$ suficientemente grande a derivada de $x(t)$ satisfa\%

$$
x^{\prime}(t)|>c \lambda| x(t-\tau) \mid>c \lambda \alpha>0
$$


Em virtude de (2.25) isso leva a uma contradiçäo, o que conclui a prova.

Juntando as informaçoes dos lemas 2.2 .3 e 2.2 .4 temos imediatamente o seguinte terolema.

Teorema 2.2.3 Seja fuma funçäo näo-decrescente e suponhamos que as segaintes condicöes estejam satiufeitas:

(i) $(2.19)$;

(ii) para k suficientemente grande, temos (2.25);

(iii) existem constantes positivas $\lambda, A, Q_{1}, Q_{2}, c \in r \in[0, \tau]$ tais que

$$
A\left(Q_{1}+Q_{2}\right)<1, \quad \lambda|x<| f(x) \leq A|x|, \quad x \neq 0
$$

é para $t$ suficientemente grande,

$$
\begin{gathered}
\int_{t}^{t+\tau} p(s) d s \leq Q_{1}, \quad \int_{t-\tau}^{t-r} p(s+\tau) d s \leq Q_{2} ; \\
p(l) \geq c
\end{gathered}
$$

Entäo, toda solusär de (2.9) e (2.10) tende a zero quando $t \rightarrow \infty$.

Scguindo agora a linha de investigaçâo de [14] é possívol estabelecermos os resultados abaixo.

Lema 2.2.5 Suponhamos que

$$
\sum_{k=1}^{\infty} b_{k}^{-}<\infty .
$$

onde $b_{k}^{-}=\max \left\{b_{k}, 0\right\}$ eque existem constantes positivas $A, B, C, D, \alpha e r \in[0, \tau]$ tais que $D<A, \quad A(x<1, \quad B(\tau-r) \leq \alpha$;

$$
D|x|<|f(x)|<A_{1} x \mid, \quad \text { se } \quad x \neq 0,
$$


e para t suficientemenle grande valcm

$$
\int_{l-7}^{l-r} p(s+\tau) d s \leq \alpha, \quad p(t+\tau) \geq B, \quad p(t) \leq C .
$$

Entäo. Loda soluça nào-oscilatória de (2.9) e (10.10) tende a zero quando $t \rightarrow \infty$.

Demonstração: Tomemos um inteiro positivo $N$ tal que as hipóteses sobre a função $p$ do lema estejam satisfeitas para todo $t \geq t_{N}$ e

$$
\sum_{k-\Lambda}^{\infty} b_{k}^{+}<1-\alpha A
$$

Scja $x(t)$ uma solução năo-oscilatória de (2.9) e (2.10) e suponhamos, sem perda de generalidade, que $x(t)>0$ para $t \geq t_{N}$. Vamos considerar a seguinte funç̧ão

$$
y(t)=x(t)-\int_{l-\tau}^{t \cdot r} p(s-\tau) f(x(s)) d s-\sum_{t_{N} \leq t_{k}<t} b_{k}^{-} x\left(t_{k}\right) .
$$

Primeiramente vamos provar que $\lim _{l}>\infty y(t)<\infty$.

Para $t \neq t_{k}, t \neq t_{k}+\tau, k=1,2, \cdots$, temos

$$
y^{\prime}(t)=-p(t-r+\tau) f(x(t-r))
$$

Por (2.29), podemos verificar que

$y\left(l_{k}^{+}\right)-y\left(l_{k}\right)=x\left(l_{k}^{+}\right)-b_{k}^{+} x\left(l_{k}\right)-x\left(l_{k}\right)-x\left(l_{k}\right)\left[b_{k}-b_{k}^{+} \leq 0, \quad k-N, N+1, \cdots\right.$ juntamente com o fato que $y^{\prime}(t) \leq 0$ para $t \geq t_{N}+r$, temos que $y$ é não-crescente (in $\left[l_{N}+r ; \infty\right)$.

Seja $L=\lim _{t \rightarrow \infty} y(t)$. Provemos que $L \in \mathbb{R}$. Se $L=-\infty$, entaio, como $y$ é não-crescente, por (2.29) $x(t)$ deve ser ilimitado. Assim, é possível escolher $t^{*}>t_{N}+\tau$ tal que $y\left(t^{*}\right)<0$ e $x\left(t^{*}\right)-\max \left\{x(t): t_{N} \leq t \leq t^{*}\right\}>0$. 
Logo, pelas hipótesces do lema, juntanente com a desigualdade (2.28) temos

$$
\begin{gathered}
0>y\left(t^{*}\right) \geq x\left(t^{*}\right)-\int_{t^{*}-\tau}^{t^{*}-r} p(s+\tau) f(x(s)) d s-\sum_{t_{N} \leq t_{k}<l^{*}} b_{k}^{+} x\left(t_{k}\right) \\
\geq x\left(t^{*}\right)-A x\left(t^{*}\right) \int_{t^{*}-\tau}^{t^{*}-r} p(s+\tau) d s-x\left(t^{*}\right) \sum_{k=N}^{\infty} b_{k}^{+} \\
\geq\left[1-A \alpha-\sum_{k=N}^{\infty} b_{k}^{\dagger}\right] x\left(l^{*}\right)>0
\end{gathered}
$$

o que é uma contradiçào. Portanto $L \in \mathbb{R}$.

Mostremos agora que $\lim _{t \rightarrow \infty} x(t)=0$. De fato, como

$$
y^{\prime}(t) \leq-B D x(t-r), \quad l \geq l_{N}, l \neq t_{k}, t \neq t_{k}+\tau
$$

podemos integrar do $t_{N}$ a $t$ ambos os membos desis desigualdade

$$
\begin{array}{r}
B D \int_{t_{N}}^{t} x(s-r) d s \leq-\int_{l_{N}}^{t} y^{\prime}(s) d s=y\left(t_{N}^{+}\right)+\sum_{t_{N}<t_{k}<t}\left[y\left(t_{k}^{\prime}\right)-y\left(t_{k}\right)\right]-y(t) \\
\leq y\left(t_{N}^{+}\right)-y(t) \leq y\left(t_{N}^{+}\right)-L_{i} \quad
\end{array}
$$

então $\int_{t_{N}}^{\infty} x(s-r) d s<\infty$, e portanto $x \in L^{1}\left(\left[t_{N}, \infty\right), \mathbb{R}\right)$. Assim, de $(2.29)$

$$
\lim _{t \times \infty} x(t)=L+\sum_{k=N}^{\infty} b_{k}^{+} x\left(t_{k}\right) \in \mathbb{R}
$$

já que $\lim _{t \rightarrow \infty} \int_{t-\tau}^{t-r} p(s+\tau) f(x(s)) d s=0$.

Mais ainda, esse limite deve ser 0 , pois caso contrário teríamos que $x \notin L^{\perp}$.

Concluindo esta seção, podemos enunciar o seguinte teorema que decorre dos lemas 2.2 .3 e 2.2 .5 .

Teorema 2.2.4 Suponhamos que (2.19) esteja satisfeito e que existam constantes positivas $\alpha, A, B, C, D$ er $r \in[0, \tau]$ tais que

$$
D|x|<|f(x)<A| x \mid . \quad A(C \tau+\alpha)<1
$$


e para t suficientemente grande valem

$$
\int_{t-\tau}^{l \cdots r} p(s+\tau) d s \leq a, \quad p(t+\tau) \geq B, \quad p(t) \leq C
$$

Entia, toda soluça de (i.9) (2.10) tende para zero quando $t \rightarrow \infty$.

\subsection{Alguns resultados para um problema planar}

Nesta scção, vamos buscar condições para que um certo problema planar apresente solução oscilatória. Vamos tratar inicialmente do caso sem impulso.

Consideremos a equação diferencial planar com retardamento

$$
x(t)=p(t) F(x(t-\tau))
$$

onde $x=\left(x_{1}, x_{2}\right) \in \mathbb{R}^{2}, F=\left(F_{1}, F_{2}\right)$ é uma funçào de classe $C^{3}$ de $\mathbb{R}^{2}$ em $\mathbb{R}^{2}$ : $\tau>0$ constante e $p:[0, \infty) \rightarrow[0, \infty)$ contínua.

Vamos acrescentar a $F$ as seguintes hipóteses

$$
x_{2} F_{1}\left(x_{1}, x_{2}\right)>0, \quad \text { se } x_{2} \neq 0 \text { e } x_{1} F_{2}\left(x_{1}, x_{2}\right)<0, \text { se } \quad x_{1} \neq 0 .
$$

Problemas somelhantes a este já foram estudados em [1] c [2]. Neste caso o espaço de fase considerado e $C\left([-\tau, 0], \mathbb{R}^{2}\right)$ com a noma do supremo.

Definiçāo 2.3.1 Uma soluçà de (2.33) é oscilatória se pelo menos uma componente näo tem sinal constante no futuro. Caso conlrário, a solucäo é chamada não-oscilatória. 
Lema 2.3.1 $S$

$$
\liminf _{t \rightarrow \infty} \mu(l)>0
$$

e se, para am dado $M>0$, existem constantes positivas $\delta(M)$ e $\eta(M)$ tais que

$$
\begin{aligned}
& \liminf _{\left|x_{1}\right|>\infty}\left|F_{2}\left(x_{1}, x_{2}\right)\right| \geq \delta(M), \quad \text { se } \quad\left|x_{2}\right| \leq M, \\
& \liminf _{\left|x_{2}\right| \rightarrow \infty}\left|F_{1}\left(x_{1}, x_{2}\right)\right| \geq \eta(M), \quad \text { se } \quad\left|x_{1}\right| \leq M
\end{aligned}
$$

Entäo, toda soluçäo núo trivial de (2033) é oscilatória.

Demonstração: Scja $x(t)$ mma solução de (2.33) e mostremos que cla é oscilatória. Suponhamos que a(t) seja não-oscilatória e assim, sem perda de generalidade, podemos considerar $x_{1}(t)>00 x_{2}(t)>0$ para todo $t>t^{*}-\tau$. Entáa por $(2.34), x_{1}(t)>0$ e $x_{2}(t)<0$ para $t \geq t^{*}$ ou seja, $x_{1}(t)$ c crescente e $x_{2}(t)$ é decrescente en $t$, para $t \geq t^{*}$.

Vamos mostrar que $x_{2}(t)$ troca de sinal. Se a solução $x(t)$ permancesse o tempo todo em $Q_{1}$ então $x(t)$ poderia convergir para um ponto de $\mathbb{R}^{2}$, o qual seria um ponto de equilíbrio e isso não pode ocorrer.

Outra possibilidade seria a de $x(t)$ tender assintoticamente para uma reta horizontal. Isso também năo pode ocorrer: já que, para t suficientemente grande,

$$
\dot{x}_{2}(t)=-p(t)\left|F_{2}\left(x_{1}(t-\tau) \cdot x_{2}(t-\tau)\right)\right| \leq-c \delta(M)<0,
$$

onde $M=x_{2}\left(t^{*}\right)$ e c ć uma constante tal que, para t suficientemente grande, $p(t) \geq$ c. Segue do Trorema do Valor Médio que $x_{2}(t)$ troca de sinal, ou scja, a solução $x(t)$ fatalmente entra em $Q_{2}$. Repetindo o mesno processo para a solução en $Q_{2}$, é possível provar que $x_{1}(t)$ troca de sinal fazerılo com que a solução entre em $Q_{3}$ e assim sucessivanunte. Isso tudo implica que a $a(t)$ deve ser oscilatória.

Consideremos agora a equação diferencial planar com retardamento

$$
\dot{x}(t)=p(t) F(x(t-\tau)), \quad t \neq t_{k}
$$


sujeita à condição de impulso radial, dada por

$$
\Delta x\left(t_{k}\right)=b_{k} x\left(t_{k}\right)
$$

onde os $b_{k}$ são constantes o os instantes de impulso satisfazem $0<t_{1}<t_{2}<\cdots<$ $t_{k}<\cdots$ c $\lim _{k \rightarrow \infty} t_{k}=\infty$. As hipóteses sobre as funções $F$ e $p$ são as mesmas estabelecidas no início desta seção.

Neste caso, definimos o espaço $P C_{\sigma}=\left\{\dot{\phi}=\left(\phi_{1}, \phi_{2}\right):[\sigma-\tau, \sigma] \rightarrow \mathbb{R}^{2}\right.$, contínua no conjunto $[\sigma-\tau, \sigma] \backslash\left\{l_{k}, k=1,2, \ldots\right\}$ e nos pontos $l_{k}$ do intervalo $[\sigma-\tau, \sigma]$, $\phi_{1}$ e $\phi_{2}$ podem ter descontinuidades de primeiro tipo e continuas à esquerda

A definição de solução para o caso planar é similar ao caso escalar.

Definiçāo 2.3.2 Uma funçùo $x(t)=\left(x_{1}(t), x_{2}(t)\right)$ é uma solução de (2.37) e (2.38) sulisfazendo

$$
x(t)=\phi(t), \quad t \in[\sigma-\tau, \sigma]
$$

$\phi \in P C_{\sigma}$, no intervalo $[\sigma ; a)$, se $x:[\sigma, a) \rightarrow \mathbb{R}^{2}$ satisfaz (2.39) e

A) para $\ell \in(\sigma, a) . t \neq t_{k}, t \neq t_{k}+\tau, x(t)$ c continua, diferenciável $e$ satisfa $(2.37)$ :

B) para $t_{k} \in[\sigma, a), x\left(t_{k}\right), x\left(t_{k}\right)$ existem o $x\left(t_{k}^{-}\right)=x\left(t_{k}\right)$ esatisfaz (2.38);

C) para. $\in(\sigma, a), t \neq t_{k}, t=t_{k}+t \tau, x(t)$ é conlínua e $\dot{x}\left(t^{*}\right), \dot{x}\left(t^{-}\right)$ existem.

Ainda de forma intciramente análoga ao caso cscalar, pode-se obter a existência e unicidade de solıção para o problema impulsivo planar.

Observação 2.3.1 Para o caso impulsivo vale observar que uma solução $x(t)$ de (2.37) e (2.38) é oscilatória se, e só sc. pelo menos uma das seguintes condiçós estäo satisfeitas. 
a) pelo menos uma das componentes $x_{i}$ tem zeros arbilrariamente grandes, isto $\dot{e}$, dado $T>0$ suficiontemente grande existe $l^{*} \geq T$ tal que $x_{i}\left(t^{*}\right)=0$;

b) pelo menos uma das componentes $x_{i}$ salisfaz a seguinte condiçäo: para qualquer $K$ sufuciememente grande existe um inteiro $k \geq K$ tal que $x_{i}\left(t_{k}^{+}\right) x_{i}\left(t_{k}\right)<$ 0 .

Observação 2.3.2 Vale notar que o exemplo contido na observaçă 2.2.1 pode ser adaptado para mostrar qur: o problema impulsivo planar pode possuir soluçao näo-oscilatória.

A partir de agora daremos condiçöes para que existam soluções oscilatórias.

Lema 2.3.2 Suponhamos que (2.34), (2.35) e(2.36) estejam satisfeitas e?

$$
\begin{gathered}
\lim _{k \rightarrow \infty} b_{k}=0, \\
l_{k}-l_{k-1}>\tau>0, \\
p(t) \geq c,
\end{gathered}
$$

onde c á uma constante positiva, e

$$
\left|F_{j}(x)\right| \geq \lambda|x|, \quad j=1,2
$$

com $\lambda>0$ constante. Entäo, toda soluçäo năo trivial de (2.37) e (2.38) é oscilatória.

Demonstração: Seja $x(t)=\left(x_{1}(t), x_{2}(t)\right)$ uma solução de $(2.37)$ e (2.38). So existir $t_{1}^{*}>0$ tal que $x_{1}(l)>0$ e $x_{2}(t)>0$ para $t>t_{1}^{*}-\tau$, pela hipótese (2.34) temos que $x_{1}(t)$ é una funçäo crescente e $x_{2}(l)$ ó decrescente para $t \neq t_{k}$, $k=1,2, \cdots$ e $t>t_{1}^{*}$. 
Mostremos que, para $k$ suficientemente grande $x_{2}\left(t_{k}^{\prime}\right)>x_{2}\left(t_{k-1}^{+}\right)$. De lato, por (2.38) segue que

$$
x_{2}\left(t_{k}\right)=\frac{1}{b_{k}+1} x_{2}\left(t_{k}^{+}\right)
$$

Assim,

$$
x_{2}\left(t_{k}\right)>\frac{1}{b_{k}+1} x_{2}\left(t_{k+1}\right) . \quad \text { e } x_{2}\left(t_{k+1}\right)>\frac{1}{b_{k} 11} x_{2}\left(t_{k+1}\right) .
$$

Portanto.

$$
x_{2}(t)>\frac{1}{b_{k}+1} x_{2}\left(t_{k+1}\right) \quad t \in\left(t_{k-1}, t_{k-1}\right)
$$

Agora, vamos comparar o quanto a função $x_{2}(t)$ decresce am $\left(t_{k}, t_{k+1}\right)$ em relação ao quanto ela salta em $t_{k-1}$.

Para $t \in\left(t_{k}, t_{k+1}\right)$, vale

$$
\left|\dot{x}_{2}(t)\right| \geq c \lambda\left|x_{2}(t-\tau)\right|>c \lambda \frac{1}{b_{k}+1} x_{2}\left(t_{k+1}\right)
$$

então

$$
\left|\dot{x}_{2}(t)\left(t_{k+1}-t_{k}\right)\right|>c \lambda \frac{1}{b_{k}+1} x_{2}\left(t_{k+1}\right) \tau
$$

logo, para k: suficientemente grande, como $b_{k} \rightarrow 0$ quando $k>\infty$

$$
c \lambda \frac{1}{b_{k}+1} x_{2}\left(t_{k+1}\right) \tau \geq b_{k+1} x_{2}\left(t_{k-1}\right)=\Delta x_{2}\left(t_{k+1}\right)
$$

o que implica $x_{2}\left(t_{k}^{+}\right)>x_{2}\left(t_{k+1}^{+}\right)$.

Assim, existe $M>0$ tal que $x_{2}(t) \leq H$, para $t>t_{1}^{*}$. Então, para $t \neq l_{k}$, suficientemente grande, por (2.35) temos

$$
\dot{x}_{2}(t)=-p(t)\left|F_{2}(x(t-\tau))\right| \leq-r \delta(M)<0 .
$$


Portanto, para $k$ suficientemente grande vale

(i) $x_{2}\left(l_{k}^{+}\right)-x_{2}\left(t_{k+1}\right) \geq c \delta(M) \tau$

0

(ii) $\quad b_{k} M \leq \frac{1}{2} c \delta(M) \tau$

De (i) o (ii) temos

$x_{2}\left(t_{k}^{-}\right)-x_{2}\left(t_{k+1}^{-}\right)=x_{2}\left(t_{k}^{+}\right)-x_{2}\left(t_{k \mid 1}\right)-\Delta x_{2}\left(t_{k+1}\right)$

$$
\geq c \delta(M) \tau-\Delta x_{2}\left(t_{k+1}\right) \geq c \delta(M) \tau-M b_{k+1} \geq \frac{1}{2} c \delta(M) \tau
$$

para $k$ suficientemente grancle, o que implica que $x_{2}(t)$ fatalmente se toma negativa.

Agora, se $x_{1}(l)>0$ e $x_{2}(l)<0$ para todo $t>t_{2}^{*}-\tau$, repetindo a mesma idéia realizada acima, prova-se que $x_{1}$ troca de sinal, e assim sucessivamente. 


\section{Capítulo 3}

\section{Equações Diferenciais Diferença Impulsivas}

\subsection{Introdução}

Estamos interessados em encontrar soluçóes periódicas para um problema planar impulsivo. Sabemos que existem resultados, por exemplo [12], que estabelecem a existência de solıção periódica não trivial quando um certo tipo de impulso é introduzido à equaçào

$$
x(t)-a F(x(t-1))
$$

onde $x=\operatorname{col}\left(x_{1}, x_{2}\right) \in \mathbb{R}^{2}, F: \mathbb{R}^{2} \rightarrow \mathbb{R}^{2}$ c $\alpha>0$.

O objetivo deste capítulo é apresentar condiçóes para que a equação

$$
\dot{x}(t)=F(x(t-r))
$$

sujeita a una condição de impulso mais geral que a tratada em [12] possua solução periódica não trivial, onder $r$ ć um númoro real positivo, $x=\operatorname{col}\left(x_{1}, x_{2}\right) \in \mathbb{R}^{2} \mathrm{e}$ $F: \mathbb{R}^{2} \rightarrow \mathbb{R}^{2}: F=\operatorname{col}\left(F_{1}, F_{2}\right)$ é contínua o $C^{\prime}$ numa vizinhança da origem. Apresentaremos também alguns exemplos. 
Essals hipóteses sobre a funçà $F$ serâo admiticlas cm todo este capítulo.

\subsection{Estudo de periodicidade}

Sejam $a>0, c>0$ constantes e

$$
l_{a, c}=\left\{\left(x_{1}, x_{2}\right) \in \mathbb{R}^{2}: \quad x_{1}-a, \quad x_{2} \geq a\right\}
$$

Consideremos a equaçào acima sujeita à uma condição de auto-sustentação impulsiva:

$$
\begin{aligned}
& \dot{x}(t)=F(x(t-r)) \\
& x(t-) \in l_{a, c} \Rightarrow x(t)=x(t+)=\left(a, \gamma\left(x_{2}(t)\right)\right)
\end{aligned}
$$

onde $\gamma:[c, \infty) \rightarrow[b, c]$ é uma função contínua que satisfaz $\gamma(c)=c$.

Lma mancira de estabelecer um resultado de existência de solução periódica para o sistema (3.1) é definir um operaklor de retorno en algum suennjunto $K$ de $C$, o estabelecer a existencia de ponto fixo não trivial. A condiçäo de impulso (3.2) introduz descontinuidades de primeiro tipo nas soluçoes, fato que exige algumas adaptações a este procedimento clássico. Utilizaremos o Teorema 1.0.1 para provarmos a existència de ponto fixo de um certo operador de retorno.

Inicialmente, definiremos solução para o problema impulsivo descrito acima.

Devido as descontinuidades que aparecom nas soluçöes, vamos considerar o conjunto $G=G\left([-r, 0], \mathbb{R}^{2}\right)$ que co o espaço das funçóes reguadas, definidas em $[-r, 0]$ com imagem em $\mathbb{R}^{2}$, veja capítulo 1 . Definamos o subconjunto $D$ de $G$ por: $\varphi \in D$, se e só so:

(i) $p \in G$;

(ii) pé contínua à direita em $[-r, 0)$; 
(iii) $\varphi$ tem no máximo $u m$ ponto de descontinuidade $t_{0} \in[r, 0]$, o qual satisfaz

$$
\varphi\left(l_{0}\right)=\left(a, \varphi_{2}\left(t_{0}\right)\right) \in l_{t, r:} \Rightarrow \varphi\left(l_{0}\right)=\left(a, \gamma\left(\varphi_{2}\left(t_{0}\right)\right)\right)
$$

Suponhamos que a condiçăo inicial

$$
x_{0}=\varphi \in D
$$

Como se trata de un problema autonomo podemos, sem perda de generalidade, fixar $t_{0}=0$.

Definição 3.2.1 Uma soluçăo do problema (3.1), (3.8) e (3.3) E uma funçŭo $x:[-r, \omega) \rightarrow \mathbb{R}^{2}: \omega>0$, tal que

(i) $\left.x\right|_{[-r, 0]}=\varphi$;

(ii) $x(t)=\varphi(0)+\int_{0}^{t} F(x(s-r)) d s, \quad l>0$ e enquanto $x(t-) \notin l_{a, c}$

(iii) se té tal que $x(\ddot{t}-)=\left(a, x_{2}(\bar{t})\right) \in l_{a, c}$ entäo

$$
x(t)=\left(a, \gamma\left(x_{2}(i)\right)\right)+\int_{t}^{t} F(x(s \cdots r)) d s, \quad l>t e c n q u n l_{0} x(l \cdots) \notin l_{a, c} .
$$

Nào é dlifícil ver cule dado $\varphi \in D$ existe uma única soluçāo de (3.1), (3.2) e (3.3) definida em $[-r, \infty)$.

Vamos assumir, en todo o capítulo. algumas hipóteses sobre a função $F$ : 
(H1) $x_{2} F_{1}(x)>0$, se $x_{2} \neq 0$ o $x_{1} F_{2}(x)<0$, se $x_{1} \neq 0$.

(H2) Existo uma constante $M>0$ tal que $|F(x)| \leq M$, para qualquer $x \in \mathbb{R}^{2}$.

(H3) Dado $L>0$, existem constantes positivas $\delta(L)$ e $\eta(L)$ tais que

$$
\begin{aligned}
& \liminf f_{\left.\left|x_{1}\right|-\infty\right)} \mid F_{2}(x) !>\delta(L) . \quad \text { se }\left|x_{2}\right| \leq L, \\
& \liminf f_{\left|x_{2}\right| \cdot ; \infty}\left|F_{1}(x)\right|>\eta(L), \quad \text { se }\left|x_{1}\right| \leq L .
\end{aligned}
$$

Se denotarmos $\frac{\partial F_{1}}{\partial x_{2}}(0)=\delta_{1}$ e $\frac{\partial F_{2}}{\partial x_{1}}(0)=\delta_{2}$, a hipótese $(\mathrm{H} 1)$ implica $\delta_{1},-\delta_{2} \geq$ 0. Vamos supor tanbém que

$$
\delta=\sqrt{-\delta_{1} \delta_{2}}>0
$$

A fin de aplicarmos o Trorema 1.0 .1 precisamos inicialmente considerar um conjunto fechado, limitado e convexo.

Seja entäo, o subconjunto de $C=C\left([-r, 0], \mathbb{R}^{2}\right)$, fechado e comvexo dado por

$$
\begin{aligned}
K= & \left\{\phi \in C \mid \phi(\theta) \in Q_{3},-r \leq \theta \leq 0, \phi_{1}(-r)=0, \phi_{2}(-r) \leq 0\right. \\
& \left.\phi_{j} \text { näo-crescentes em }[-r, 0] \text { para } j=1,2 \text { e são } M \text {-Lipschitzianas }\right\} .
\end{aligned}
$$

Queremos demonstrar o seguinte teorema que garante a existência do solıção periódica para o problemra estabolecido neste capítulo.

Teorema 3.2.1 Se as hipóteses (H1), (HQ) e (H3) estiverem satisfeitas, cntão existe um conjunto limitado $K^{\prime} \subset K$ de modo que o problema (3.1) e (3.2) tem uma soluáa periódica não trivial $x(.: \phi), \phi \in K^{\prime}$ com perhodo maior que $3 r$. 
Para provarmos este fato é necessário construirmos $11 \mathrm{~m}$ operador completamente contínuo, $A: K \rightarrow C$, mostrar que existe um subconjunto limitado de $K$ de forma que esse subconjunto seja invariante por $A$ e finalmente mostrar que este operador tem um ponto fixo que não é o zero. Para definirmos tal operador precisamos do alguns lemas.

Lema 3.2.1 Consideremos o problema (3.1), (是的) com as hipóteses (H1), (HQ) (Hs). Entäo riste uma funcia continua $t_{1}: K \backslash\{0\} \rightarrow[0, \infty)$ bal que $x_{2}\left(t_{1}(\phi) ; \phi\right)=$ $0, x_{1}\left(t_{1}(\phi) ; \phi\right)<0, x(t ; \phi) \in Q_{3}$ para $0 \leq t \leq t_{1}(\phi)$ e $x(l ; \phi) \in \operatorname{int}\left(Q_{4}\right)$ se $t_{1}(\phi)<t<t_{1}(\phi)+\delta$. para algum $\delta>0$ suficientemente pequeno.

Demonstração: Para $t>0, \phi \in K$, c encuanto a solução $x(., \phi)$ permanecer en $Q_{3}$, a hipótese (H1) implica que a semi-órbita positiva $\{x(l ; \phi), l>0\}$ não pode convergir para a origem. Note também que $x(t ; \phi)$ não porle convergir para um ponto $P_{0} \neq(0,0)$ de $Q_{3}$ quando $t \rightarrow \infty$, pois o 0 é o único ponto de equilíbrio da equação (3.1).

Sabemos também que essa scmi-órbita näo pode convergir assintoticamente para una semi-reta horizontal, pois se isso ocorrer, ou seja, $x_{2}(t ; \phi) \rightarrow \bar{x}_{2} \leq 0$ quando $l \rightarrow \infty$, então $\mid x_{1}(l ; \phi) \rightarrow \infty$ e por (H3), para $l$ suficientemente grande,

$$
\dot{x}_{2}(l)=H_{2}(x(l-r)) \geq \delta(L)>0
$$

o que implica que, passado um tempo finito, $x_{2}(t ; \phi)$ se torna positivo. Assim, a órbita cruza transversalmente o semi-eixo $\left\{\left(x_{1}, 0\right) ; x_{1}<0\right\}$ num instante $t_{1}=$ $t_{1}(\phi)$. A continuidade de $t_{1}$ segue de argumentos clássicos envolvendo o Teorema da Continuidade em relaçăo às condiçōes iniciais, voja, por excmplo, Lema 3.7 de 
[11], Lema 5.50115 .6 de [1.], Lema 3.3 de [12].

Podemos também considerar as funçóes $t_{j}$ definidas em $K \backslash\{0\}, j-2,3,4$, descritas abaixo.

Soja $t_{2}: K \backslash\{0\} \rightarrow[r: \infty), \phi \longmapsto l_{2}(\phi)$ : contínua de forma que $x_{1}\left(t_{2}(\phi) ; \phi\right)=$ $0, x_{2}\left(t_{2}(\phi) ; \phi\right)>0, x(t ; \phi) \in Q_{4}$ para $t_{1}(\phi) \leq t \leq t_{2}(\phi)$ e $x(t ; \phi) \in \operatorname{int}\left(Q_{1}\right)$ se $t_{2}(\phi)<t<t_{2}(\phi)+\delta$, para algum $\delta>0$ suficiententente pequeno. A existéncia e a continuidade de to podem ser demonstradas de modo inteiramente análogo à existência e continuidade do $/ 1$.

Soja agrora $/_{3}: K \backslash\{0\} \rightarrow[2 r, \infty), \phi \longmapsto t_{3}(\phi)$, de forma que:

-Se $\phi \in K \backslash\{0\}$ é tal que $x(t ; \phi)$ não sofre impulso, ou seja, a solução da equação passando por $\phi$ não encontra a semi-reta $l_{a, c^{\prime}}$ então $t_{3}(\phi)$ satisfaz: $x_{2}\left(t_{3}(\phi) ; \phi\right)=$ $0, x_{1}\left(t_{3}(\phi) ; \phi\right)>0, r(t ;) \in Q_{1}$ para $t_{2}(\phi) \leq t \leq t_{3}(\phi)$ e $x(t ; \phi) \in \operatorname{int}\left(Q_{2}\right)$ se $t_{3}(\phi)<t<t_{3}(\phi)+\delta$, para algum $\delta>0$ suficientemente pequeno.

- Se $\phi \in K \backslash\{0\}$ é tal que $x(t ; \phi)$ sofre impulso, isto é, existe $t_{0} \operatorname{com} x\left(t_{0}-; \phi\right) \in$ $l_{a, c}$, então vamos considerar três situaçòes:

1) Se $x\left(l_{0}, \phi\right) \in Q_{1}$, então $l_{3}(\phi)$ satisfaz as condiçoes acima.

2) Se $x\left(t_{0}, \phi\right) \in Q_{2}$ e a solução é tal que $x(t ; \phi) \notin Q_{1}$ para $l_{0}<t<t_{0}+r$, entào clefininos $t_{3}(\phi)=t_{0}$ : que é o instante de impulso.

3) Se $x\left(t_{0} ; \phi\right) \in Q_{2}$ e a solução $x(t ; \phi) \in Q_{1}$ para algum $t$ com $t_{0}<t<t_{0}+r$, definimos

$$
t_{3}(\phi)=\max \left\{t \in\left[t_{0} \cdot t_{0}+r\right] ; x_{1}(t ; \phi)>0 x_{2}(t, \phi)=0\right\} .
$$

Podemos observar pela definição que t/3 nãa é uma função contínua, de $\phi$.

Finalmente, seja $t_{4}: K \backslash\{0\} \rightarrow[2 r, \infty), \phi \longmapsto t_{4}(\phi)$ satisfazendo $x_{1}\left(t_{1}(\phi) ; \phi\right)=$ $0, x_{2}\left(t_{4}(\phi) ; \phi\right)<0, x(t ; \phi) \in Q_{2}$ para $t_{3}(\phi) \leq t \leq t_{4}(\phi)$ e $x(t ; \phi) \subset \operatorname{int}\left(Q_{3}\right)$ se $t_{4}(\phi)<t<t_{4}(\phi)+\delta$, para algum $\delta>0$ suficientemente pequeno. A existencia 
de $t_{1}(\phi)$ segue de argumentos análogos à prova de $t_{1}$.

Lema 3.2.2 A funçăo to definida acima é contínua.

Demonstração: $S c \phi C K, \phi \neq 0$ e $x(t ; \phi)$ nà sofre impulso para $t \in\left[0, t_{1}(\phi)\right]$

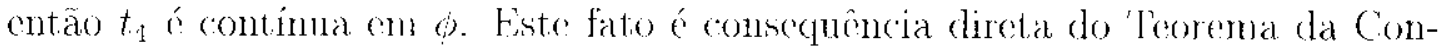
tinuidade com relagão às condiçoes iniciais, como já foi observado quando tratamos da continuidade do $t_{1}(\phi)$.

Seja $K_{i} \subset K \backslash\{0\}$ o conjunto das $\phi \in K$ tais que $x(t ; \phi)$ sofre impulso jara $t \in\left[t_{2}(\phi), t_{3}(\phi)\right]$.

Se $\phi \in K_{i}$ denotemos por $t_{i}(\phi) \in\left[t_{2}(\phi), t_{3}(\phi)\right]$ o momento de impulso de $x(l ;()) . \quad A$ aplicação $\phi \in h_{i} \longmapsto t_{i}(\phi) \in \mathbb{R}$ é contínua pelo mesmo motivo que $t_{1}, t_{2}$ são contininas.

Seja agora $C_{0} \subset C$ tal que $C_{0}=\left\{x_{t+r}(. ; \phi) ; \phi \in K_{i}, t \in t_{i}\left(K_{i}\right)\right\}$. A aplicaçà $\phi \in K_{1} \longmapsto x_{t_{i}(\phi)+r}(.: \dot{\phi}) \in C_{0}$ é contínua. De fato, se consideramos o problemal sem inpulso, isto é, dado somente por

$$
\dot{y}(t)=F(y(t-r))
$$

denotando por $y(t: \phi)$ a solução dessa equacão passando por $\phi \in K_{i}$, temos que a aplicação $\phi \in K_{i} \longmapsto y_{t_{i}(\phi)-r}(; \phi) \in C$ para $t \in\left[t_{i}(\phi), t_{i}(\phi)+r\right]$ é contínua. Assim, para o caso com impulso, podenos escrever

$$
x_{t_{i}(\phi)-r}(. ; \phi)=y_{t_{i}(\phi)+r}(. ; \phi)-\left(0, \gamma\left(y_{2}\left(t_{i}(\phi)\right)\right) .\right.
$$

Como y é contínua segue-se imediatamente que a aplicação

$$
\phi \subset \Lambda_{i} r x_{l_{i}(0)+\cdots}(. ; \phi) \in C_{0}
$$

ćcontínua. 
Agora, para cada $\phi \in C_{0}$, soja $t^{\prime}(\phi)$ tal que $a_{1}\left(t^{\prime}: \phi\right)=0, x_{1}(t ; \phi)>0$ para $t \in\left[0, t^{\prime}\right] \odot x_{2}\left(t^{\prime} ; \phi\right)<0$. Notemos que, se $\phi=x_{t i(b)-r}(; \psi)$, com $\psi \in K_{i}$, cntáa $l^{\prime}(\phi)=t_{4}\left(t^{\prime}\right)$. Como $t^{\prime}: C_{0} \longrightarrow \mathbb{R}$ é também contínua segue que to restrita a $K_{i}$ é contínua.

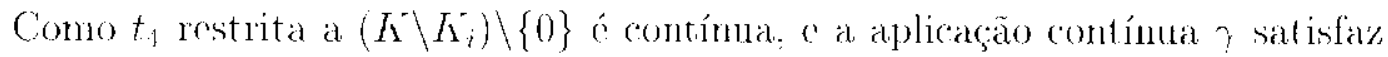
$\gamma(c)=r$ segue-se que ti é contínua em $K \backslash\{0\}$.

Podemos então definir a scguinte função contínua

$$
\tau: K \backslash\{0\} \rightarrow(3 r, \infty)
$$

tal que

$$
\tau(\phi)=l_{4}(\phi)+r, \quad \tau(\phi)>0
$$

Definamos agora o operador de retorno em $K$,

$$
A \phi= \begin{cases}x_{i(\phi)}(\cdot \varphi), & \text { se } \phi \in K \backslash\{0\} \\ 0, & \text { se } \phi=0\end{cases}
$$

Precisamos mostrar que este operador é completamente contínuo.

Para isto, vamos precisar do seguinte lema. cuja denonstragão ć uma adaptação da prova do Lema 3.5 de [12].

Lema 3.2.3 Seja $G \subset C$, aberto; $0 \in G$. Existe uma vizmhança $V \subset C$ de o tal que se $\phi \in V \cap K, \phi \neq 0$, tem-sex $x_{f}(: \phi) \subset G$ part todo $0 \leq \ell \leq t_{1}(\phi)+r$.

Demonstração: É suficiente provar que dado $G^{\prime} \subset \mathbb{R}^{2}$ aberto, $0 \in G^{\prime}$; existe: uma vizinhança $V$ de $0 \mathrm{~cm} C$ tal que se $\phi \in V \cap K, \phi \neq 0$, então $x(t ; \phi) \in G^{\prime}$ para $-r \leq t \leq t_{1}(\phi)+r$. 
Admitanos que vale a afimagão acina para $\left.t \in:-r, t_{1}(\phi)\right]$ e mostremos que vale para $t \subset\left[t_{1}(\phi) ; t_{1}(\phi)+t\right]$.

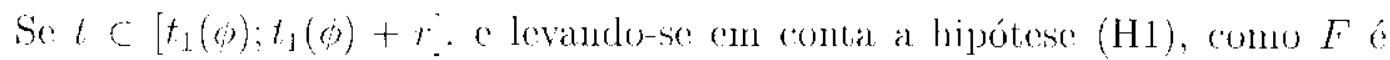
contínua o $F(0)=0$, então dade $t>0$, existe $\delta>0$ tal que se $|x(t-r ; \phi)|<\delta$ temos

$$
\begin{aligned}
& -r \leq F_{1}(x(t-r)) \leq 0 \\
& 0 \leq F_{2}(x(t-r)) \leq \epsilon .
\end{aligned}
$$

Logo

$$
\begin{gathered}
-\epsilon \leq \dot{x}_{1}(t) \leq 0 \\
0 \leq \dot{x}_{2}(t) \leq \epsilon
\end{gathered}
$$

o que implica $0 \leq x_{2}(l ; \phi) \leq r\left(r-c r-\delta \leq x_{1}(l ; \phi) \leq 0\right.$ para $l \subset\left[t_{1}(\phi) ; t_{1}(\phi)+r\right]$. Portanto, fica verificada a afirmação acima.

Mostremos agora que existe uma vizinhança $V$. como descrito no enunciado do lema, tal que $x(t ; \phi) \in G^{\prime}$, para $-r \leq t \leq t_{1}(\phi)$. com $\phi \in V \cap K$. Suponhamos, por absurdo, que isto năo scja válido. Assim, existe um retangulo

$$
R=\left\{\left(x_{1}, x_{2}\right) \in \mathbb{R}^{2} ; \quad-l<x_{1}<\delta_{1}:-\delta_{2}<x_{2}<m\right\}
$$

onde $\delta_{1}, \delta_{2}, l, m$ sào todas constantes positivas, tal que, para alguna sequiencia $\left(\phi_{n}\right), \phi_{n} \in K \backslash\{0\}: \phi_{n} \rightarrow 0$ quando $n \rightarrow x$, existe mat seqünencia (t $t_{n}$ ) com $-r \leq t_{n} \leq t_{1}\left(\phi_{n}\right)$ satisfaymdo $x\left(l_{n}: \omega_{n}\right) \in \partial R, x\left(t: \phi_{n}\right) \in R$ patra $-r \leq t \leq t_{n}$; $n=1,2,3, \ldots$

Se denotarmos $x_{n}(t):=x\left(t ; \phi_{n}\right) ; x_{n}^{i}(t)-x_{i}\left(t: \phi_{n}\right), i=1,2$, teremos $x_{n}(t) \in$ $[-1,0)^{\circ} \times\left[-q_{n}, 0\right],-r \leq t \leq t_{n}$, onde $q_{n}=\left\|\phi_{n}\right\| \rightarrow 0$ (quando $n \rightarrow \infty$. 
Consecuentemente, $x_{1}\left(t_{n} ; \phi_{n}\right)=-l, n=1,2,3, \ldots$ A continuidade de $F_{1}$ co fato de quo $F_{1}\left(x_{1}, 0\right)-0$ implicam que

$$
\lim _{x_{2} \rightarrow 0^{-}} F_{1}\left(x_{1}, x_{2}\right)=0
$$

uniformemente em $x_{1}$ em $[-1,0]$.

Agora, tomando uma sequéncia $\left(\tau_{n}\right)$, de modo que, $0<\tau_{n}<t_{n}, x_{n}(t) \in$ $\left[-\frac{l}{2}, 0\right] \times\left[-q_{n}, 0\right]$ se $-r \leq t \leq \tau_{n} \operatorname{com} x_{1}\left(\tau_{n} ; \phi_{n}\right)=\cdots \frac{l}{2}, n=1,2, \ldots$ Como $q_{n} \rightarrow 0$, quando $n \rightarrow \infty$, temos que $x_{1}\left(\tau_{n}-r ; \dot{\varphi}_{n}\right)<-\frac{1}{3}$, pois $\left|\dot{x}_{n}^{\perp}(l)\right|=\left|F_{1}\left(x_{n}(t-r)\right)\right|$ pode ser feito arbitrarianente pequeno tomando-se $n$ suficientemente grande. Portanto, para qualquer $t \in\left[\tau_{n}-r_{1} l_{n}\right]$, vale $x_{1}\left(t ; \phi_{n}\right)<-\frac{l}{3}$.

Scjam $\sigma>0$ e $n_{0}$ un inteiro positivo tais que $\left|F_{2}(x)\right|>\sigma$ se $x \in \mathbb{R}^{2}:\left|x_{1}\right| \geq \frac{l}{3}$ e $n \geq n_{0}$ implica $\left|F_{1}\left(x_{n}(t)\right)\right|<\sigma$ e $q_{n}<\frac{l}{2}$.

Como $x_{1}\left(t ; \phi_{n}\right)$ é estritamente decroscente e $x_{2}\left(t: \phi_{n}\right)$ é estritanente crescente para $t \in\left[\tau_{n}, t_{n}\right], x_{n}(t)-x\left(l ; \phi_{n}\right)$ co o grálico da função $x_{2}=x_{n}^{2}\left(x_{1}\right)$.

Portanto para $\tau_{n} \leq t \leq t_{n}$ temos $-l \leq x_{1}(t) \leq-\frac{l}{2}$ e vale

$$
\frac{d x_{2}}{d x_{1}}-\frac{\left|F_{2}\left(x_{n}(t-r)\right)\right|}{\left|F_{1}\left(x_{n}(t-r)\right)\right|}>1
$$

o que é unia contradiçăo.

Lema 3.2.4 Seja $G(C$ C aberto: $0 \in G$. Existe uma vizinhança $Y C$ de o tal que se $\phi \in V \cap K, \phi \neq 0$, tem-se $x_{t}(: 0) \subset G$ para todo $0 \leq t \leq \tau(\phi)$.

Demonstração: Vão há perda de generalidade en considerar $C \subset B_{\delta} \subset C$ tal que $0<\delta<\sqrt{a^{2}+c^{2}}$, onde $B_{\delta}$ representa a bola aberta em $C$. isto $c$, $B_{\delta}=\{\phi \in C ;\|\phi\|<\delta\}$. Assim, devido as simetrias de nossas hipóteses este 
resultado ce uma consecpuencia do Lema 3.2.3.

Dado $\xi>0$, podenos definin para cada $\xi$ o conjunto limitado $K_{x i}$ de $C$ por

$$
\begin{aligned}
& K_{\xi}=\left\{\varphi \in C \mid \varphi(\theta) \in Q_{3},-r \leq \theta \leq 0, \varphi_{1}(-r)=0,-\xi \leq \varphi_{2}(-r) \leq 0\right. \\
& \left.\varphi_{j} \text { não crescentes em }[-r .0] \text { para } j=1,2 \text { e são } M \text {-Lipschitzianas }\right\}
\end{aligned}
$$

Notemes cue o conjunto $\Lambda$ näo é limitade, nass devido à forma do impulso, o Lema 3.2 .5 a seguir, garante a existencia de um subconjunto limitado de $K$, para o qual voltam as soluçós que dole parten. Mais precisamente, este conjunto ó invariante polo operador $A$.

Lema 3.2.5 Fxiste $\xi>0$ tal que $A K \subset K_{\xi}$ e, portanto, $K_{\xi}$ é invariante por $A$.

Demonstração: Seja $N$ um subconjunto de $C$ tal que $\phi=\left(\phi_{1}, \phi_{2}\right) \in N$ se, e só se, $\phi_{1}$ e $\phi_{2}$ são M-Lipschitziandas o satisfaz uma das seguintes altermativas:

a) $0 \leq b_{1}(-r) \leq a\left(\phi_{2}(-r)-0, \phi_{1}(0)\right.$ a näro decrescente c $\phi_{2}(0)$ ć não crescente om $[-r \cdot 0]$;

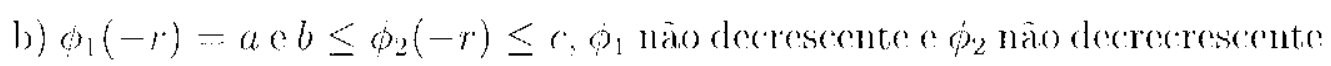
cm $\left[-r, \theta_{0}\right]$ ? não cresconte om $\left[\theta_{0}, 0\right]$, para algum $\theta_{0},-r \leq \theta_{0}<0$.

Definamos

$$
\begin{aligned}
T_{M}=\left\{\left(x_{1}, x_{2}\right)\right. & \left.\in \mathbb{R}^{2} ; \quad 0 \leq x_{1} \leq a+r M ;-r M \leq x_{2} \leq 0\right\} \\
\cup\left\{\left(x_{1}, x_{2}\right)\right. & \left.\in \mathbb{R}^{2} ; \quad a \leq x_{1} \leq a+r M ; b-r M \leq x_{2} \leq r+r M\right\} .
\end{aligned}
$$

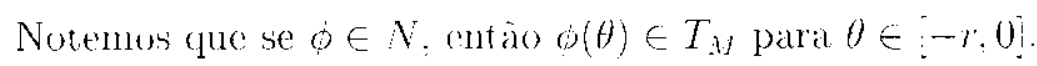


$N$ é um subconjunto compareto de $C$; já que $T_{M}$ é um subronjunto compacto de $\mathbb{R}^{2}$ a as funçós de $N$ são $M$-Lipschitzianas.

Vamos clefinir a seguinte funçäo:

$$
f: N \rightarrow \mathbb{R}
$$

tal que a cada funçào $\phi \in N$ associamos $f(\phi)=x_{2}\left(t^{*} ; \phi\right)$, onde $t^{*}=t^{*}(\phi)$ c 0 instantre em que a solução $x(t: \phi)$ da equação (3.1) passa de $Q_{2}$ para $Q_{3}$.

Precisamos primeiramente mostrar que existe uma funcão $t^{*}: N \rightarrow[0, \infty)$ tal (110: dado $\phi \in N, x_{1}\left(t^{*} ; \phi\right)=0, x_{2}\left(t^{*} ; \phi\right)<0$ ex $x(t ; \phi) \in \operatorname{int} Q_{3}$ para $t^{*}<t<t^{*}+\delta$ para qualquer $\delta>0$ prequeno, onde $t^{*}=t^{*}(\phi)$.

Por adaptaçoes dos argumentos da prova do Lema 3.2.1, referenta à existoncia e continuidade de $t_{1}: k \backslash\{0\} \rightarrow[0, \infty)$, pode-se garantir a existência e a continuidade da funçăo $t^{*}: N \rightarrow[0, \infty)$

Portanto a função $f$ c contínua o satisfaz $f(\phi)<0$ para todo $\phi \in N$, $\log o$ existe $\xi>0$ tal que $f(\theta) \geq-\xi$, para todo $\phi \in N$. Como as soluçoes, $x(1 ; \psi)$, com \&: $\in K \backslash\{0\}$ do problema (3.1) ( (3.2) devenu passar por $T_{A}$, conclúnos que. para toda $b \in K, \xi \neq 0$, vale $x_{\tau(\eta)}(, ; b) \in K_{\xi}$. Notemos que $f(b)=x_{2}\left(t_{1}(t) ; \psi\right)$. com $\phi=x_{1}(; t)$ para algum $t>0 e t \in K$.

De agora em diante consideraremos sempre $K_{\xi}$, oncle $\xi$ satisfaz: $A K_{\xi} \subset K_{\xi}$. A possibilidade dessa escolla é garantida polo lema anterior.

Lema 3.2.6 A aplicaçäo $A: K_{\xi} \rightarrow K_{\xi}$ definida por

$$
\begin{gathered}
A \phi=x_{\tau(\phi)}(. \therefore) \quad \phi \neq 0 \\
A 0=0
\end{gathered}
$$

é completamente contínua. 
Demonstração: So $\phi_{0} \in K_{\xi} \backslash\{0\}$ entào a continuidade de $A$ ar $\phi_{0} 6$ consequencia innediata da continuidade de $l_{1}$ a do Teorema da Contimuidade das soluçoes em relaçăo dis condiçoes iniciais.

A continuidade de $1 \mathrm{em} \phi_{0}=0$ singue inediatamente do Lema 3.2.t.

Seja agora $\bar{B}_{\eta}$ a bola fochada em $C$ de centro em 0 o raio $\eta$ e seja $V=K_{\xi} \cap \bar{B}_{\eta}$. Então, pela construçáo de $K_{\xi}$

$$
A V=\left\{x_{\tau(\phi)}(. ; \phi): \phi \in V \backslash\{0\}\right\} \cup\{0\} \subset K_{\xi}
$$

logo, $A V$ é limitado. Como os elementos do conjunto $h_{\xi}$ são funções cujas componentes são M-Lipschitzianas segue imediatamente a equicontinuidade de Al. Portanto A é completamente contínua.

Podemos observar que a solução mula ce uma solução periódica para o problema dado. Para descartarmos tal solução devemos mostrar cue cla é ponto cjetivo do operador de retorno $A$.

Observação 3.2.1 Para provurmos que zero é ponto ejelivo de 1 basta analisarmos o caso scm impulso. De fato, suponhamos que o zero seja ponto ejetivo para o problema sem impulso, ou soja, existe uma vizinhançaberta $G \subset K$ de zero tal que para cada $\phi \in G \cap K, \phi \neq 0$, existe um inteiro $m=m(\phi)$ tal que. $\bar{A}^{m} \phi \notin G \cap K$, onde A é operador de retorno de $K$ om $K$ para o problema dado pela equaçào $\dot{x}(t)=F(n(t-r))$, sem a condiçäo de impulso.

Se tal winhanca cucontra a semi-reta $l_{a, r}$, basta tomarmos uma vizinhansa menor $G^{\prime}$ contida em $G$ de forma que $G^{\prime}$ näo encontre $l_{n, c}$. Em $G^{\prime}$ o operador A coincide com o operador A do problemo impulsino. logo o mesmo inteiro $m$ satisfaz a definiça 1.0.1 para A. Agora, se a vizinhanç $G$ nao encontra a semirela $l_{a, c}$, podemos considerar a própria vizinhança $G$ para o operador A definido $\sin K_{\xi}$. 
Consideraremos unicanente o caso sem impulso.

Lincarizando a equaça e utilizando a decomposição do espaço $C$ descrita no Capítulo 1, vamos cmunciar o lema a seguir que fornece condição suficientes para a cjetividade da origem de $u$ m cento operador de retorno. A prova pode ser encontrada em [1], Isema 5.2.

Lema 3.2.7 Suponhamos que as seguintes condiçoes estejam satisfeitas:

(i) existe um auto-valor $\lambda$ da equaçăo linearizada linear com $\Re(\lambda)>0$.

(ii) existe um subconjunto $K$ convexo e fechado de $C, 0 \in K$, e uma funçäo contínua $\tau: K \backslash\{0\} \rightarrow[\rho, \infty), \rho>0$, tal que a aplicacäo $A: K \rightarrow C$, dada por

$$
A \phi= \begin{cases}x_{\tau(\phi)}(\therefore \phi), & \text { se } \phi \neq 0 \\ 0, & \text { se } \phi=0\end{cases}
$$

é completamente contínua e $A K \subset K$.

(iii) Se $0<a<L$, para algum $L \in \mathbb{R}$,

$$
\inf \left\{\left\|\Pi_{\lambda}\left(x_{t}\right)\right\|: x_{t}-x_{t}(. ; \phi), \theta \in K,\|\phi\| \geq a, 0<t<\tau(\phi)\right\}>0 .
$$

(iv) Dado $G \subset C$ aberto $0 \in G$, existe uma vizmhanga $V$ de 0 em $C$ tal que $x_{l}(., \phi) \subset G$ se $\phi \subset \mathrm{l} \cap K, \phi \neq 0,0 \leq t \leq \tau(\phi)$.

Entäo () é um ponto ejetivo de A.

Retornando ao nosso caso, como o nosso operador A leva limitados om limitados, os mesmos argumentos dos lemas 3.2 .6 e 3.2 .4 garantem as hipóteses (ii), (iv) respectivamento para o caso sem impulso. Resta-nos mostrar (i) e (iii). 
Para provarmos (i) precisanos analisal a cenacăo característica do problema linearizado. A equaça linearizada de (3.1) em tormo da origem ó dada por

$$
\dot{y}(t)=B y(t-r)
$$

onde

$$
B=\left(\begin{array}{ll}
\frac{\partial F_{1}}{\partial x_{1}}(0,0) & \frac{\partial F_{1}}{\partial x_{2}}(0,0) \\
\frac{\partial F_{2}}{\partial x_{1}}(0,0) & \frac{\partial F_{2}}{\partial x_{2}}(0,0)
\end{array}\right)=\left(\begin{array}{ll}
0 & \delta_{1} \\
\delta_{2} & 0
\end{array}\right)
$$

A cquaçào $\dot{y}(t)=B y(t-r)$ ć um caso particular da equação $\dot{x}(l)-L x_{t}$ com

$$
L(\phi)=\int_{-r}^{(0)}[\operatorname{d\eta }(\theta)] \phi(\theta)=B \phi(-r)
$$

onde $\eta(\theta), 0 \in-r, 0]$ a matriz de ordem 2 definida por

$$
\eta(\theta)- \begin{cases}-B, & \text { se } \theta=\cdots r \\ 0, & \text { se }-r<\theta \leq 0 .\end{cases}
$$

A equação característica de (3.9) é dada por

$$
\Delta(\lambda)=\lambda I-\int_{-r}^{0} e^{\lambda \theta} d \eta(\theta)=\lambda I-B e^{-\lambda r}
$$

onde I representa a matriz identidade de ordem 2. Logo, a equação característica tem a seguinte forma 


$$
\lambda^{2} e^{2 \lambda r}=\delta_{1} \delta_{2}<0
$$

A análise desta eruaçào característica está contida nos lemas a seguir e cstá estritamente relacionada com o estudo feito em [11], onde ć tratado uma versão da equaçào (3.10).

Lema 3.2.8 Existe exalamente uma raiz $\lambda=a+$ bi da equaça (3.10) com $0<b<\frac{\pi}{2 r}, a>0$ e cla é simples.

Demonstração: Srja $\lambda=a+b i$ tal que $0<b r<\frac{\pi}{2}$, então

$$
\Re\left(\lambda e^{\lambda r}\right)=e^{a r}(a \cos b r-b \operatorname{sen} b r)
$$

e por (3.10) temos que $\lambda e^{\lambda t}=\delta i$, lembrando que $\delta=\sqrt{-\delta_{1} \delta_{2}}$, assim

$$
a \cos b r-b \operatorname{sen} b r=0
$$

o que inplica

$$
\text { (I) } a=b \tan b r>0 \text {. }
$$

Considerando agora a parte imaginária de $\lambda e^{\lambda r}$. temos

$$
(I I) \quad e^{a r}(a \operatorname{sen} b r+b \cos b r)=\delta \text {. }
$$

Substituindo (I) (m (II) obtemos

$$
\delta=\Lambda(b)=\frac{b e^{b r \tan b r}}{\cos b r}
$$

mitaio 


$$
\begin{gathered}
\frac{d \Lambda}{d b}=\frac{e^{b r \tan b r}\left[\cos b r+2 r b \tan b r \cos b r+b^{2} r^{2} \sec b r \cos b r\right]}{\cos ^{2} b r} \\
=e^{b r \tan b r}\left(1+2 b r \tan b r+b^{2} r^{2} \sec ^{2} b r\right) \sec b r \\
=r^{b r \tan b r}(1+b r \tan b r)^{2}+b^{2} r^{2} \sec b r>0 .
\end{gathered}
$$

além disso

$$
\lim _{b \rightarrow 0+} \Lambda(b)=0 \quad \lim _{b \rightarrow \frac{\pi}{2 r}-} \Lambda(b)=\infty
$$

As caracteristicas da função $\Lambda$ garantem (1ue podemos definir mivocamente b) (omo função de $\delta, 1<\delta<\infty$. Pela equaçăo (I) temos que a pode ser considerada também cono uma funçào de $\delta$, encuanto $0<b r<\frac{\pi}{2}$, mais ainda $\lambda$ é diferenciável (om respeito a $\delta$.

Seja $\lambda_{0}$ raiz de $(3.10)$. Iosde que $\lambda_{0} \neq-\frac{1}{r}$, temos

$$
\frac{d}{d \lambda}\left(\lambda^{2} e^{2 \lambda r}-\delta_{1} \delta_{2}\right)_{\lambda=\lambda_{0}}-2 \lambda_{0} e^{2 \lambda_{0} r}\left(1-\lambda_{0} r\right) \neq 0
$$

o que implica que a rais $\lambda_{0}$ é simples.

O lema anterior garante a existencia de un autovalor com parte real positiva o que impliea o ítem (i) do Lema 3.2.7. Resta-nos somente provar (iii). Para isto vamos precisar do seguinte resultado.

Lema 3.2.9 Suponhamos que as hipóteses (II1). (H2) e (H3) eslejam satisfeitas. Seja $d>0$. Fxiste $D>0$ tal que se $\phi \in \Gamma$ e $\|\phi\| d$ enlao $x_{1}\left(t_{1}(\phi) ; \phi\right) \leq-D$.

Demonstração: É suficiente mostral que dado $k>0$, existe $\delta>0$ tal que se

$$
R_{\gamma, k}-\left\{\left(x_{1}, x_{2}\right) \in \mathbb{R}^{2} ;-\delta \leq x_{1} \leq 0,-h \leq x_{2} \leq 0\right\},
$$


$\operatorname{com} \phi \in K, \mathrm{e} \phi(0) \notin R_{\delta, k} \operatorname{cnt} \tilde{a} o x_{1}\left(t_{1}(\phi), \dot{\phi}\right)<-\delta$.

Assim, dado $k>0$, definamos

$$
M_{\delta}=\sup \left\{\left|F_{2}(x)\right| ;-\delta \leq x_{1} \leq 0,-M r-k \leq x_{2} \leq-k / 2\right\}
$$

para todo $\delta>0$. Como $F_{2}$ ć contínual e $F_{2}\left(0, x_{2}\right)=0$ para todo $x_{2} \in \mathbb{R}$, 1, mos que $\lim _{\delta \rightarrow 0}+M_{\delta}=0$.

Seja agora

$$
\eta=\min \left\{\mid F_{1}(x) ; x=\left(x_{1}, x_{2}\right),-1 \leq x_{1} \leq 0,-M r-k \leq x_{2} \leq-k / 2\right\} \geq 0 .
$$

Consideremos entào $\delta$ satisfazendo $0<\delta<\min \{1, k / 2\}$ e $M_{\delta}<\eta$.

Seja $\phi \in K$ tal que $\phi(0) \notin R_{\delta, k}$ e suponhamos que exista $l_{0}>0$ tal que $x_{2}\left(l_{0} ; \phi\right)=k,-\delta \leq x_{1}\left(l_{0} ; \phi\right) \leq 0$. Se não cxistir tal $l_{0}$ nä́o há o cue provar.

Encuanto $x(t ; \phi) \in R_{\delta, k}$ com $x_{2}(t ; \phi) \leq-k / 2$ para $t \geq t_{0}$, temos

$$
\frac{-k}{2} \geq x_{2}(t-r) \geq-M r-k \text {. }
$$

Portanto. $\left|F_{2}(x(t-r ; \phi))\right| \leq M_{\delta}<\eta$, de acordo com (3.12). Além disso,

$$
\left|F_{1}(x(t-r ; \phi))\right| \geq \eta,
$$

de acordo com (3.13). Logo o vetor $\dot{x}(t ; \phi)$ satisfaz

$$
\frac{\left|\dot{x}_{2}(l ; \phi)\right|}{\left|\dot{x}_{1}(t ; \phi)\right|} \leq \frac{\eta}{\eta}=1
$$

Portanto, lembrando que $\delta<\frac{-k}{2}$, existe $t \geq t_{0}$ tal que

$$
x_{1}(\bar{t}, \phi)=\cdots \delta, \quad x_{2}(\bar{t} ; \phi) \leq-k / 2
$$

e assim concluimos que $x_{1}\left(t_{1}(\phi), \phi\right) \leq-\delta$. 
Devido às simetrias das hipóteses: podemos repetir os argmmentos acima c provar a scrguinte proposição.

Proposição 3.2.1 Suponhamos que as hipóteses (III), (H2) e (H3) estejam satisfeitas. Seja $d>0$. Existem $D_{2}, D_{3}>0$ tal que se $\phi \in K \in\|\phi\| \geq d$ entäo $x_{2}\left(t_{2}(\phi) ; \phi\right) \geq D_{2} e x_{1}\left(t_{3}(\phi) ; \phi\right) \geq D_{3}$

Considerando o problema linearizado na origem, sendo $E$ o gerador infinitesimal do semigrupo, podemos decompor o espaço $C$, como foi mencionado no Capítulo 1 .

Pelo Lema 3.2.8, existe um único autovalor $\lambda$ tal que $\Re \lambda>0$, para $0<$ $\Im \lambda<\frac{\pi}{2 r}$, que é simples, portanto, o auto-espaço generalizado $P_{\lambda}=N\left(\lambda I-E^{*}\right)$ é 1-dimensional.

Se ué uma soluçäo nào trivial de $\Delta(\lambda) u=0$ com $\Delta(\lambda)=\lambda I-B e^{-\lambda r}$, e vetor linha v ć uma soluçào náa trivial de $v \Delta(\lambda)=0$, entäo $\rho(l)=e^{\lambda \ell} u, \ell \in[-r, 0]$ e $\sigma(s)=v e^{-\lambda s}, s \in\left[0, r\right.$ geram $N(\lambda I-E) \circ N\left(\lambda I-E^{*}\right)$, respectivamente.

Assim, se $\phi \in C$, a sua componente $\phi^{\prime \prime}$ rm $P_{\lambda}$ é dada por

$$
\phi^{P_{\lambda}}=\langle\sigma, \phi\rangle \rho
$$

onde a forma bitineras (. : .) édedinida no Capítulo 1 por (1.1).

Lema 3.2.10 Se as hipóteses (H1). (HD) e (H.3) estiverem satisfeitus, 4e $\bar{M}$ é tal que, $x(l ; \phi)$ näo sofre impulso para $\phi \in K,\|\phi\| \leq M \quad t \in\left[-r, t_{4}(\phi)\right]$, então existe $N>0$ tal que

$$
|x(t ; \phi)| \leq N \quad \text { para } \quad t \in\left[-r, t_{4}(\phi)\right]
$$


Demonstração: Scja $\phi \in h .\|\phi\| \leq \bar{M}$ de modo que $x(t ; \phi), t \in\left[-r, t_{4}(\phi)\right]$ nào

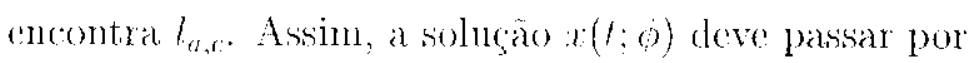

$$
\left\{\left(x_{1}, x_{2}\right) \in \mathbb{R}^{2} ; 0 \leq x_{1} \leq a, x_{2}=0\right\}
$$

ou por

$$
\left\{\left(x_{1}, x_{2}\right) \in \mathbb{R}^{2} ; x_{1}=a, 0 \leq x_{2} \leq c\right\}
$$

Por (H1) e (H3) deve existir $k_{1}>0$, independente de $\phi$, tal que $x_{1}\left(t_{3}(\phi) . \phi\right) \leq$ $k_{1}, t_{3}(\phi)$ definida atrás para o caso sem impulso.

Consideremos o conjunto

$$
K_{1}=\left\{\eta \in C \quad \eta(\theta) \in Q_{2},-r \leq \theta \leq 0,0 \leq \psi_{1}(-r) \leq k_{1}, \psi_{2}(-r)=0\right.
$$

$\psi_{1}$ é crescente e $\psi_{2}$ é decrescente em $[-r: 0]$ e $t_{j}$ para $j=1,2$ săo $M$-Lipschitz. $\}$.

Notemos que $K_{1}$ é um subconjunto compacto do espaço $C$, pois seus elementes são funçoes limitadas e $M$-Lipschitzianas. Novamente, usando as hipóteses (H1) e (II3) podemos garantir a existencia de $t_{1}: K_{1} \backslash\{0\} \rightarrow \mathbb{R}$ satisfazendo, $x(t ; \psi) \in$ $Q_{2}: t \in\left[-r, \hat{t}_{1}(\varphi)_{i}: x_{1}\left(\hat{t}_{1}(\omega), \varphi\right)=0, x_{2}\left(t_{1}(\varphi), \omega\right)<0, x(t, \psi) \in \operatorname{int} Q_{3}\right.$ para $i_{1}(t)<t<\hat{t}_{1}(b)-\delta$, para algum $\delta>0$. Pelo Teurena da Continuidade em relaça as condiçoes iniciais temos que t.l $(b)$ é contínna, cntão a aplicaça $\psi \in K_{1} \backslash\{0\} \mapsto x_{2}\left(t_{1}(\omega), t\right)$ ó contínua e portanto assume nuínimo, ou seja, existe $k_{2}>0$ tal que $x_{2}\left(\hat{t}_{1}(\psi), \psi\right) \geq-k_{2}$ para toda $\psi \in K_{1} \backslash\{0\}$. Isso implica que existe um número $C_{1}>0$ tal cque

$$
|x(t ; \phi)| \leq C_{1}, \quad \forall \theta \in K_{1}, \quad t \in\left[-r, \hat{t}_{1}(\psi)+r\right] .
$$

Notemos também que existe $\psi \in K_{1}$ tal que $\psi=x_{t_{3}\langle\phi\}+r}(., \phi), \phi \in K$, lombrando que $x(t ; \phi)$ não sofre impulso.

Procedendo de mancira análoga. prova-se que existem constantes positivas $k_{3}$

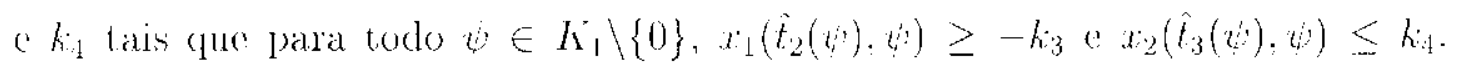
Sogue innediatamente a existencia de $C_{2}>0$ tal que 


$$
|x(t ; \psi)| \leq C_{2} . \quad \forall \subset K_{1} \backslash\{0\}, \quad t \in\left[-r, \hat{l}_{1}(\psi)\right]
$$

onde as funçoes $i_{j}: K_{1} \backslash\{0\} \rightarrow[0, \infty), j=2,3,4$ säo contínuas e satisfazem:

(i) $x(t ; \varphi) \in Q_{3}, t \in\left[t_{1}(\varphi), t_{2}(\varphi)\right], x_{1}\left(t_{2}(\varphi), \varphi\right)<0, x_{2}\left(t_{2}(\varphi), \varphi\right)=0, x(t, \varphi) \in$ int $Q_{3}$ para $t_{2}(\varphi)<t<\hat{t}_{2}(\varphi)+\delta$, para algum $\delta>0$;

(ii) $x(t ; \varphi) \in Q Q_{1}, t \in\left[t_{2}(\varphi), t_{3}(\varphi)\right], x_{1}\left(t_{3}(\varphi), \varphi\right)=0, x_{2}\left(t_{3}(\varphi), \varphi\right)>0, x(t, \varphi) \in$ $\operatorname{int} Q_{4}$ para $\hat{t}_{3}(\varphi)<t<\hat{t}_{3}(\varphi)+\delta$, para algum $\delta>0$;

(iii) $x(t ; \varphi) \in Q_{1}, t \in\left[t_{3}(\varphi), t_{1}(\varphi)\right], x_{1}\left(l_{4}(\varphi), \varphi\right)>0, x_{2}\left(\hat{t}_{4}(\varphi), \varphi\right)=0, x(t, \varphi) \in$ int $Q_{1}$ para $t_{1}(\varphi)<t<i_{1}(\varphi)+\delta$, para algum $\delta>0$.

Tomando $N^{\prime}=\max \left\{\bar{M}+M r, C_{j}+M r\right\} j=1,20 N=\sqrt{2} N^{\prime}$, tomos

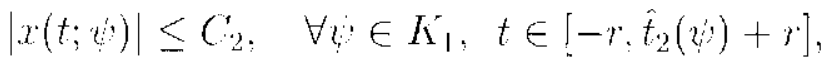

enquanto a soluçào nào encontra a semi-reta $l_{a, c}$.

Lema 3.2.11 Seja d e $\bar{M}$ tal que $0<d<M$. Entäo

$\inf \left\{\|\|_{\lambda} x_{l}\left\|; x_{l}=x_{l}(; \phi) ; \phi \in K \backslash\{0\}: d \leq\right\| \phi \| \leq \bar{M} ; 0 \leq t \leq \tau(\phi)\right\}>0$.

Demonstração: Soja $v=(1:-i \mu)$ onde $\mu=\sqrt{-\delta_{1} / \delta_{2}}>0$, entăo $v$ satisfaz $v \Delta(\lambda)-0, o \sigma(s)-(1,-i \mu) r^{-\lambda s}:$ coms $s \in[r, 0]$ ó um elemento de $C^{*}$, cntào

$$
\left\langle\sigma, x_{l}\right\rangle=x_{1}(l)-i \mu x_{2}(t)+\int_{-r}^{0} \int_{-r}^{\theta}(1,-i \mu) e^{\lambda(\theta \iota)} d \eta(\varsigma) x(t+\theta) d \theta
$$

Calculando a integral de Sticltjes, 1 mos

$$
\left\langle\sigma, x_{1}\right\rangle=x_{1}(l)-i \mu x_{2}(l)+\int_{-r}^{0} e^{\lambda(\theta-r)}\left[\delta_{1} x_{2}(t+\theta)-i \mu \delta_{2} x_{1}(t+\theta)\right] d \theta .
$$


Mostremos primeirancente que

$m=\inf \left\{\left\|\prod_{\lambda} x_{t}\right\| ; x_{l}=x_{t}(. \dot{\phi}) ; \phi \in K \backslash\{0\}: d \leq|\vdots \phi| \leq \bar{M}, 0 \leq t \leq t_{1}(\phi)+r\right\}>0$.

Vamos supor: por contradiçäo. que $\mathrm{m}=0$. Fntão existem scequencias $\phi_{n} \in$ $K \backslash\{0\}, \operatorname{com} d \leq \mid \phi_{n} \| \leq \bar{M}$ e $t_{n} \in\left[0, t_{1}\left(\phi_{n}\right)+r\right], n=1.2, \ldots$ tais que, se denotarmos $x^{n}(t)=x_{\ell}\left(. ; \phi_{n}\right)$ vale

$$
\mid x_{1}\left(l_{n}\right)-i \mu x_{2}\left(t_{n}\right)+\int_{r}^{0} e^{-\lambda(\theta+r)}\left[\delta_{1} x_{2}\left(l_{n}-\theta\right)-i \mu \delta_{2} x_{1}\left(t_{n}+\theta\right)\right] d \theta ! 0
$$

cuando $n \rightarrow \infty$.

$S e \lambda=a+b i$, com $a>0$ e $0<b r<\frac{\pi}{2}$. chtaro podemos ver que

$$
\begin{array}{r}
\mid x_{1}\left(t_{n}\right)+\int_{r}^{0} e^{-a(\theta+r)}\left[\delta_{1} x_{2}\left(t_{n}+\theta\right) \cos (b \theta+b r)-\mu \delta_{2} x_{1}\left(t_{n}+\theta\right) \operatorname{sen}(b \theta-b r)\right] d \theta \\
+i\left\{-\mu x_{2}\left(t_{n}\right)+\int_{-r}^{0} e^{a(\theta \cdot r)}\left[-\delta_{1} x_{2}\left(t_{n}+\theta\right) \operatorname{sen}(b \theta+b r)\right.\right. \\
\left.\left.-\mu \dot{\partial}_{2} x_{1}\left(t_{n}+\theta\right) \cos (b \theta+b r)\right] d \theta\right\} \mid \rightarrow 0
\end{array}
$$

Notemos que $0<b \theta+b r<\pi / 2, \operatorname{com} \theta \in[-r, 0]$. Como $d \leq\left\|\phi_{n}\right\| \leq \bar{M}$, entäo pelo Lema 3.2.10 temos que $\left\|x^{n}(t)\right\| \leq N$, para $t \in\left[-r, t_{1}(\phi)+r\right], n=1,2, \ldots$, onde as constantes $\bar{M}$ o $N$ säo aquelas definidas no Lema 3.2 .10 e na sua prova. Assinı: podemos afirmar que $x^{n}\left(t_{n}\right) \rightarrow\left(L_{1}, L_{2}\right)$ com $n \rightarrow \infty$; tomando uma subsegüència so necessário.

O Lema 3.2.9 garante que $\left(L_{1}, L_{2}\right) \neq\left(0\right.$, (0). Se $L_{1}<0$ e $L_{2} \leq 0$, podemos ver que $\left\{\Omega\left(\left\langle\sigma, x_{n,}\right\rangle\right)\right.$ fica negativo o uniformemente afastado do zero para $n$ grande, o que contraria $(3.15)$.

$S e L_{1}=0$ e $L_{2}<0$ (ntara $3\left(\left\langle\sigma, x_{t_{i}}\right\rangle\right)$ fica positivo e uniformemente afastado do zero para $n$ grande, o que também contraria (3.15).

Finalnnemmte, se $L_{2}>0$, então necessariamente $L_{1}<0$. Para ne grande temos 


$$
x_{1}\left(t_{n}: \phi_{n}\right)<\frac{L_{1}}{2} \quad \odot \quad x_{2}\left(t_{n} ; \phi_{n}\right)>\frac{L_{i_{2}}}{2}
$$

Vamos denotal

$$
p_{n}(\theta)=\delta_{1} r_{2}\left(l_{n}+\theta\right)-i \mu \delta_{2} x_{1}\left(l_{n}+\theta\right), \quad \theta \in[-r, 0] .
$$

P'ara ne graude $\mu_{n}(\theta)$ está no sutor

$$
\left\{\rho e^{i \alpha x} ; \rho \geq 0-\pi / 2 \leq \alpha \leq 0\right\}
$$

e a exponencial $e^{-\lambda(\theta-r)} \operatorname{com} \theta \in[-r .0]$ está no setor

$$
\left\{\rho e^{i \alpha \alpha} ; e^{-a r} \leq \rho \leq 1,-B \leq \alpha \leq 0, B \in(0, \pi / 2)\right\}
$$

Considerando o produto $e^{-\lambda(\theta+r)} p_{n}(\theta), \theta \in[-r, 0]$ para $n$ grande temos que o argumento $\alpha$ satisfaz: $-B-\pi / 2 \leq \alpha \leq 0$ com $B \in(0, \pi / 2)$, isso implica que, para $n$ grande, $\circlearrowleft\left\{e^{\lambda(\theta \cdot r)} p_{n}(\theta)\right\} \leq 0$, enta $\Im\left(\left\langle\sigma, x_{n}\right\rangle\right)$ fica negativo e afastado uniformemente do zero, assim (3.15) não pode acontecer. Portante $m>0$.

O lena segue agora por aplicacoos sucessivas desses argumentos, explorando as simptrias de nosisas hipóteses.

Com este último resultado e pela obscrvação 3.2 .1 segue que a origem é ponto ejetivo do operador $A$.

Sendo zero ponto ejetivo e juntando as conclusões dos lemas 3.2 .6 o 3.2.5. segue que todas as hipóteses do Teorema 1.0.1 estão satisfeitas tomando-se o operador $A$ como o opcrador de retorno definido cm (3.6), o conjunto $K_{\xi}$ no lugar de $K$ e o ponto ejetivo como sendo a origem. Assim, o operador A tem ponto fixo nào nulo, o que corresponde a uma soluça periódica do problema impulsivo com período maior que $3 \%$, concluindo assim a prova de 3.2 .1 . 
Observação 3.2.2 Uma soluçào periódica $x(t ; \phi), \phi \in K_{\xi} \backslash\{0\}$, ruju existen é estabelecida no Teorema 3.2.1 pode ser chamada lentamente espiralante como em [1], por ler o segumte comportamento: existem $t_{0}, t_{1}, t_{2}, t_{3}, t_{4}$ tais que?

$$
t_{1}-t_{0}>r: \quad l_{2}-l_{1}>r ; \quad t_{1}-t_{3}>r \quad t_{3}-t_{2}>0
$$

e.

$$
x(t ; \phi) \in Q_{i+2} \quad t_{i \cdot 1}<t<t_{i} i=1,2,3,4 .
$$

Em outras palavras, a órbita periódica permanece em cada quadrante, $Q_{2}, Q_{3}, Q_{1}$ por um lapso de tempo maior do que $r$.

Observação 3.2.3 O Teorema 3.9.1 garante a existencia de soluçăo periódica com periodo mator que $3 r$ para todo $\delta>0$. A seguir veremos que para centos valores de $\delta$, extste solucào periódica de amplitude pequena e perídos pequenos oriundas de bifurcaçäo de IIopf. Estas solucioes näo sofrem impulsos.

Este fato decorre da análise da equaça característica (3.10) que, após a mudança de parâmetros $\nu=\lambda r$ e $\beta=r \delta$, onde $\delta$ é dado em (3.2), se reduz a

$$
v^{2} e^{2 l^{\prime}}=-3^{2}
$$

Como as raizes l ocorrem aos pares conjugados, basta uma análise de sua distribuigáa no semi-plano superior, $\nu=a+b i$, tal que $a \geq 0$.

Os Lemas 2.1-9.6 de [11] implicam que as raízes $\nu$ de (3.16) se distruibuem no semi-plano complexo superior obedectndo as scguintes condiçoes:

1) Existe precisamente uma raiz $\nu^{\prime}$, que é simples, em cada faixa $S_{k}, k-$ $0,1,2, \ldots$, dada por

$$
S_{0}=\left\{\nu=a+b i ; 0<b<\frac{\pi}{2}\right\},
$$




$$
S_{k}=\left\{\nu=a+b i ;\left(k-\frac{1}{2}\right) \pi<b<\left(k+\frac{1}{2}\right) \pi\right\}, \quad k=1,2, \ldots
$$

2) Quando $\beta$ varia em $(0, \infty)$ no sentido crescente a raiz $\nu_{k}(\beta)=a_{k}(\beta)+b_{k}(\beta) i$ da fana $S_{k}, k=0,1,2, \ldots$ deserene uma curva tal que $a_{k}(B)$ e b $b_{k}(B)$ sac funcoes estritamente coescentes de is o alem disso.

$$
\begin{gathered}
\lim _{\beta \rightarrow \infty} a_{k}(\beta)=\infty \quad k=0,1,2, \ldots \\
\lim _{\beta \rightarrow 0} a_{0}(\beta)-0, \quad \lim _{\beta \rightarrow 0} a_{k}(\beta)=-\infty \quad k-1,2,3, \ldots \\
\lim _{b \rightarrow \infty} b_{k}(\beta)=\left(k+\frac{1}{2}\right), \quad k=0,1,2, \ldots \\
\lim _{\beta \rightarrow 0} b_{k}(\beta)=\left(k-\frac{1}{2} \pi\right) \quad k=1,2,3, \ldots \\
\lim _{\beta \rightarrow 0} b_{0}(\beta)=0 .
\end{gathered}
$$

3) Quando $\beta$ assume os valores $k \pi, k=1,2, \ldots$, as raizes $\nu_{k}(\beta)$ säo imaginárias puras da forma $\nu=k \pi i, k=1,2, \ldots$ isto é,

$$
\nu(k \pi)=k \pi i . \quad k=1,2,3, \ldots
$$

4) Para todo $\beta>0, a_{k+1}(\beta)<a_{k}(\beta)$ e $\lim _{k \rightarrow \infty} a_{k}(\beta)=-\infty$.

De acordo com (3.11) um cálculo simples leva a

$$
\frac{d \beta}{d b}>0
$$

Portanto, $\frac{d b}{d \beta}>0$ e como $b \tan b=a$ lemos 


$$
\frac{d a_{k}}{d \beta}>0, \quad k=1,2,3 \ldots
$$

Voltando aos parànctros originais, $\lambda e \delta$, as observacoos 3.2 .3 implicam que as raízes $\lambda$ tèm qualinativamente, a mesma distribuição das raízes v no plano complexo. Isto é, quando $\delta$ varia de 0 a $\propto$, a raiz $\lambda$ percorre uma das curvas parannetrizadas em $\delta$, indicadas na figura 3.1, no sentido crescente

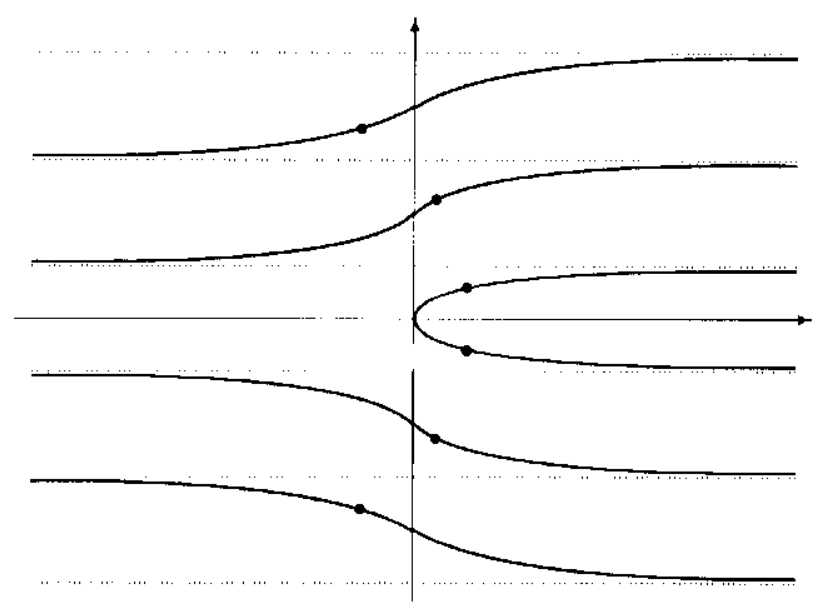

Figura 3.1: I)istribuição das raízes $\lambda$

Os valores $\delta_{k}=\frac{\beta_{k}}{r}$ correspondentes ao cruzamento das raízes $\lambda_{k}, \bar{\lambda}_{k}$ com o eixo imaginário são $\delta_{k}=\frac{k \pi}{r}, k= \pm 1 . \pm 2, \ldots$ De acordo com (3.17),

$$
\frac{d}{d \delta}\left(\operatorname{Re}\left(\lambda_{k}(\delta)\right)_{j=i_{k}} \neq 0\right.
$$

O seguinte teorema ó agora uma consequiència direta do Teorema da Bifurcaçâo de Hopf: Teorema 1.1. de [1], segundo Hale. 
Teorema 3.2.2 S: (H1) cstiver satisfeita então existe uma sequência $\delta_{1}<\delta_{2}<$ $\ldots \rightarrow \infty$ tal que a rquaço (3.1) tem uma bifurcaça de $\operatorname{Hopf}$ em $\delta_{k}, k=1,2, \ldots$ Isto é. quando $\delta$ assume um dos valores $\delta_{k}$, emana da soluçäo trivial um ramo de solucoos periódicas com perído próximo de $\frac{2 r}{k}$.

\subsection{Exemplos}

O Teorema 3.2 .1 garante a existência de soluçào periódica para o problema impulsivo (3.1) o (3.2). Uma tal solugào pode ou nâo sofrer impulsos. Neste último caso seria uma solıção periódica do problema não impulsivo (3.1). Isto sugere a questão: Nao seriam sem impulso todas as solucoes periódicas de (3.1)? Veste caso o Teorema 3.2 .1 seria um teorema relativo ao problema näo impulsivo definido apenas pela equação (3.1). Nesta seção mostraremos, através de $11 \mathrm{~m}$ exemplo. que podem efetivamente existir soluçòes periódicas impulsivas.

Consisleremos $F: \mathbb{R}^{2} \rightarrow \mathbb{R}^{2}$, definida por

$$
F^{\prime}(x)= \begin{cases}\left(x_{2},-x_{1}\right), & \text { se }|x| \leq M, \\ \frac{M\left(x_{2},-x_{1}\right)}{|x|}, & \text { se }|x| \geq M\end{cases}
$$

Não é difícil verificar que $F$ satisfaz as hipóteses (II), (H2) e (H3). Considerando agora constantes positivas a c $c$, sendo que $a \geq M r$ temos a condição de impulso dado em (3.2),

$$
x(l) \in l_{a, c} \Rightarrow x(t)=\left(a, \gamma\left(x_{2}(t)\right)\right)
$$


onde $\gamma:[c, \infty) \rightarrow[b, c]$ satisfaz as condiçoes estabelecilas no início deste capítulo. Consirleremos o conjunilo

$$
\begin{aligned}
K_{a, b, c} & =\left\{\phi \in O \mid \phi_{1}(-r)=a, b \leq \phi_{2}(-r) \leq c \phi_{1},\|\phi\|\right. \text { säo não decrescentes } \\
& \left.c \phi_{2} \text { não crescente em }[-r, 0] \text { c } \phi_{j} \text { são M-Lipschitzianas, para } j=1,2\right\}
\end{aligned}
$$

Este conjunto é focharto, limitado e convexo e, mais ainda é um subconjunto compacto de $C$ cm virtude das componentes $\phi_{j}, j=1,2$ serem $M$-lipschitzianas. Para definirmos um operador de retorno em $K_{a, b, c}$, mostremos que, se $\phi \in K_{a, b, c}$, entio a solução por $\phi, x(t ; \phi)$, tem norma $|x(t ; \phi)|$ crescente cm $t$.

De lato, dado $\phi \in K_{a, b, c}$, temos três situaçoes a considerar:

1) se $\phi(\theta) \in Q_{1}$ para todo $\theta \in[-r, 0]$.

2) se existir $\theta_{0} \in[-r, 0]$ tal cue $\phi_{2}\left(\theta_{0}\right)=0$ e $\phi_{1}\left(\theta_{0}\right)>0$.

3) se $\phi(\theta) \in Q_{2}$ para todo $\theta \in[-r, 0]$.

Utilizando os mesmos argumentos do Lema 3.2.1, podemos mostrar (1uc cxistem funçōes contínuas $\tilde{t}_{0}, \tilde{t}_{1}, \tilde{t}_{2}$ e $\tilde{t}_{3}$ satisfazendo:

$i_{0}: K_{n, b, r}^{0} \rightarrow[0, \infty)$ contínua. onde $K_{a, b, r}^{0}=\left\{\phi \in K_{a, b, c} ; \phi_{2}(-r) \geq 0\right\} \subset K_{a, b, c}$ o tal que $\iota_{2}\left(t_{0}(\phi) ; \phi\right)=0, x_{1}\left(\tilde{t}_{0}(\phi): \phi\right)>0, x(t ; \phi) \in Q_{1}$ para $0 \leq t \leq \tilde{t}_{0}(\phi)$ e $x(t ; \phi) \in \operatorname{int}\left(Q_{2}\right)$ se $\tilde{t}_{0}(\phi)<t<\tilde{t}_{0}(\phi)+\delta$. para $\delta>0$ suficientemente pequeno.

$i_{1}: K_{a, b, c} \rightarrow[0, \infty)$ contínua, tal que $x_{2}\left(\tilde{t}_{1}(\phi) ; \phi\right)<0, x_{1}\left(\check{t}_{1}(\phi) ; \phi\right)=0$, $x(t ; \phi) \in Q_{2}$ para $0 \leq t \leq \dot{t}_{1}(\phi)$ o $x(t ; \phi) \in \operatorname{minl}\left(Q_{3}\right)$ s $\tilde{l}_{1}(\phi)<l<\tilde{l}_{1}(\phi)+\delta$. para $\delta>0$ suficientemente perpueno.

$\tilde{f}_{2}: K_{a, b, c} \rightarrow[0, \infty)$ contínua, tal que $x_{2}\left(\tilde{t}_{2}(\phi) ; \phi\right)=0, x_{1}\left(\tilde{t}_{2}(\phi): \phi\right)<0$, $x(t ; \phi) \in Q_{3}$ para $0 \leq t \leq \tilde{t}_{2}(\phi)$ e $x(t ; \phi) \in \operatorname{int}\left(Q_{4}\right)$ se $\tilde{t}_{2}(\phi)<l<\tilde{l}_{2}(\phi)+\delta$, para algum $\delta>0$ suficientemente pecueno.

$$
\tilde{t}_{3}: h_{a, b_{0},} \cdots[0, \infty) \text { contínua, tal (que } x_{2}\left(\tilde{l}_{1}(\phi) ; \phi\right)>0, x_{1}\left(\tilde{l}_{3}(\phi) ; \phi\right)-0 \text {, }
$$


$x(t ; \phi) \in Q_{1}$ para $0 \leq l \leq \tilde{t}_{3}(\phi)$ o $x(t ; \phi) \in \operatorname{int}\left(Q_{1}\right)$ se $\tilde{l}_{3}(\phi)<t<\tilde{t}_{3}(\phi)+\delta$, para algnum $\delta>0$ suficientemente pexpenos.

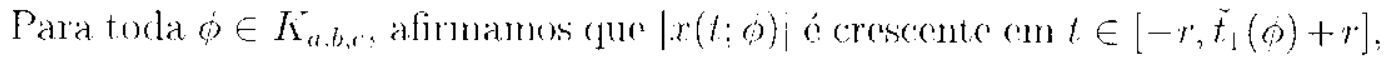
pois quando $\theta$ varia de $t-r$ a $t, x(\theta: \theta)$ percorre a órbita no sentido horário, de acordo com a Figura 3.2

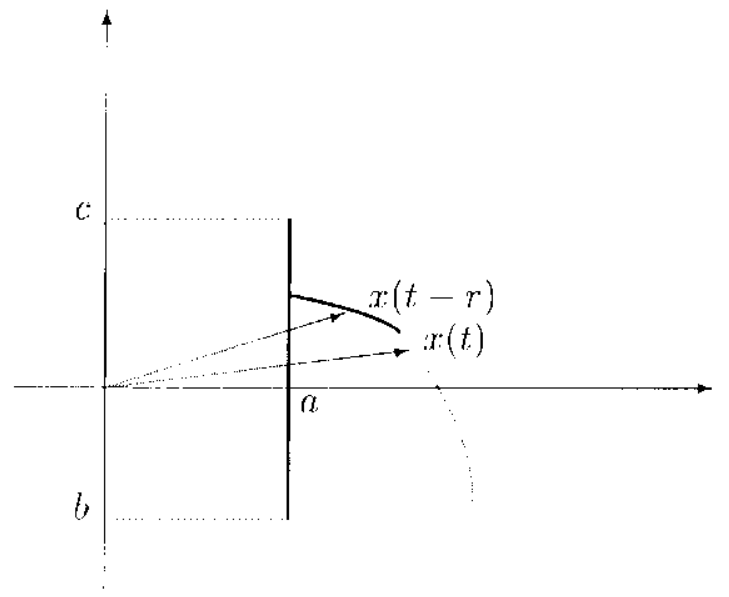

Figura 3.2: ()rientação da órbita de $x(l)$

Como $F(x)$ é o produlo de una função escalar positiva com a rotaçăo de $\pi / 2$

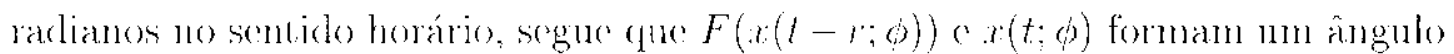
agudo, isto é:

$$
x(l ; \phi) \cdot x(l ; \phi)=x(t ; \phi) \cdot F(x(t-r ; \phi))>0
$$

e portanto $|x(t ; \phi)|$ é crescente.

Parat $>\hat{t}_{1}(6)$ i ro raciocínio é o mesmo, logo a solugão tem normal crescento até o primeiro instante de impulso.

Definamos agora a função $\tilde{\tau}: K_{a, b, c} \rightarrow(2 r, \infty)$ : onde $\tilde{\tau}(\phi)$ é o primeiro instante tal quer

$$
x_{\tau(\omega)+,+,}(: 0) \in K_{u, b, r}
$$


Votenos que esta funçăo está bem definida porque nâo existe $t>\bar{t}_{3}(\phi)$ com $0<x_{1}(t: \phi) \leq a x_{2}(t ; \phi)=0$, já que a norma da solução é arescente. Logo $\tilde{\tau}(\phi)$ fica univoramente determinado catso $x(. ;)$ sofra impulso ou náo no instante $\tilde{\tau}(0)$. A condiçăo de impulso estabelecida o o Torema da Continuidade em relaçào às condiçoes iniciais implicam que $\tilde{\tau}(\phi)$ é contínua.

Podemos, então definir

$$
\begin{gathered}
\tilde{A}: K_{a, b, c} \rightarrow K_{a, b, r} \\
\dot{A}(\phi)=x_{\bar{\tau}(\phi)-r}(\therefore \emptyset) .
\end{gathered}
$$

Como $K_{a, b, c}$ é compacto segue que á é completamente contínua. Polo Tromema de Schauder, A tem um ponto fixo, que corresponde a uma soluçào periódica não trivial do problema impulsivo.

Esta solução neccssariamente sofre impulso, pois se não sofresse teria norma crescente e, portanto, nào poderia ser periódica.

Na verdade pode-se mostrar que existem soluçóes periódicas impulsivas para campos $F$ muito mais gerais do que o do exemplo acima. Isto é, temos a seguinte proposição.

Proposiçāo 3.3.1 Se F sutisfaz as hipóteses (H1). (II) e (II3), entüo cxistem constantes $a, c>0$ tais que toda solução periódica năo trivial de (3.1) e sujeita a condiçäo de impulso

$$
x(t-) \in l_{a, r} \rightarrow x(t)=(a, \gamma(x, 2(t))),
$$

onde $l_{a, c}=\left\{\left(x_{1}, x_{2}\right) \in \mathbb{R}^{2} ; x_{1}=a, x_{2} \geq c\right\}$. impulsiva. 
Demonstração: Consideremos o conjunto $h$ definido em (3.5) e o operarlor de retorno $A: K \rightarrow K$ definido em (3.6). De acordo com os Lemas 3.2.7, 3.2.8 e 3.2 .11 e a origem é ejotiva com relagäo a $A$.

Fxiste $L>0$ e $B_{L}=\left\{\phi \in C: \| \phi_{i}^{\prime} \mid<L\right\}$ tal que $\phi \in B_{L}$, existe $n=n(\phi)$ tal que $A^{n}(\phi) \notin B_{L} \cap K$.

Pela Proposição 3.2.1, dado $L$ e j| $\dot{\|} \| L$, existe $D_{3}>0$ tal que $x_{1}\left(t_{3}(\phi): \phi\right) \geq$ $D_{3}$, onde $D_{3}$ está definido na Proposição 3.2.1. Afirmamos que para qualquer $a$, $0<a<D_{3}$, cxiste $c>0$ tal que $x(l ; \phi)$ cruza $l_{a, c}$.

Suponhamos, por contradiçáo que exista $\phi \in K$ tal que $x(t ; \phi)$ seja uma solução periódica nâo impulsiva. Então necessariamente $\|\dot{\phi}\|<L$. Portanto, existe $n=n(\phi)$ tial cure $\left\|A^{n} \phi\right\|>L$, mas isto implica que $x\left(l ; A^{n} \phi\right)$ (rura $l_{a, c}, 0$ que é uma contradição. Logo, qualquer que seja a solução periódica do problema (3.1) sujeita a condição de impulso acima é do tipo impulsiva. 


\section{Capítulo 4}

\section{Soluções periódicas contra-fluxo}

\subsection{Um caso linear}

No problema de equaços diferenciais planares tratado no capítulo 3 , a hipótese (H1) é uma versão bidimensional da condição de retro-alimentaşăo negativa classicamente formulada para o caso escalar. Esta hipótese sugere que as órbitas tenham comportamento de girar em torno da origem no sentido horário, pois o campo vetorial $F$ considerado naquele capítulo pode ser representado no diagrama absaixo:

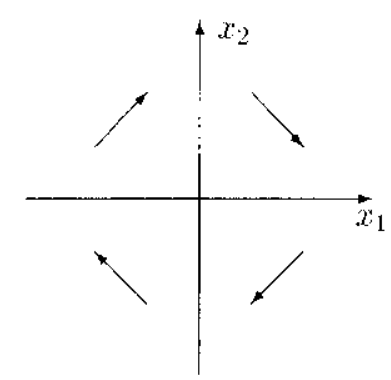

Figura 4.1: Sentido do campo

Os fatos deste capítulo demonstram. entretanto, cue podem existir soluçoes cujas órbitas giram no scntido contrário ao do campo, isto é, giram no sentido 
anti-horário em torno da origenn. Neste caso direnos que as soluçōes são contrafluxo,

So olharmos para as órbitas como caminhos no $\mathbb{R}^{2}$, uma equaçäo linear extremamente simples permite uma olservação mais contundente:

O plano porle ser coberto por una fantília de órbitas periódicas contra-fluxo e, simultancamente, por mma fannília equ gira no sentido horário (sentido do (ampo).

Consideremos o sistema de equações

$$
\begin{aligned}
& x^{\prime}(l)=\pi y(t-1) \\
& y^{\prime}(t)=-\pi x(t-1) .
\end{aligned}
$$

Este é um caso particular de (3.1). onde $F(x, y)=(\pi y,-\pi x)$.

Fazcndo $x_{1}=x$ e $x_{2}=y$, vemos que $F$ satisfaz a hipótese (II1) dada no capítulo 3.

Podemos escrever o sistema (4.1) como mma equaçä́o de segunda orelem

$$
x^{\prime \prime}(t)=-\pi^{2} x(t-2)
$$

Estannos interessados em soluçós 2-periódicas, o cue implica

$$
x(t-2)-x(t)
$$

Assim, podemos reserever o problema (4.2) como nma E.D.O.,

$$
x^{\prime \prime}(t)+\pi^{2} x(l)=0
$$

cuja solução geral $x(t)$ tem a forma

$$
x(t)=a \cos \pi t+b \operatorname{sen} \pi l,
$$


onde a e b, são constantes. Usando (4.1), obtemos

$$
y(t)=-a \cdot \operatorname{sen} \pi(t+1)+b \cos \pi(t-1),
$$

o que implica

$$
y(t)=a \operatorname{sen} \pi t-b \cos \pi l
$$

Se fizermos, por exemplo, $a=00 b=1$, termos unna solução 2-periódica da equação planlar (4.1).

$$
(x(t), y(t))=(\operatorname{sen} \pi t,-\cos \pi t),
$$

que gira no sentido anti-horário, isto é contra-fluxo.

Como o sistema é linear, temos na verdade, uma família a um parânetro or,

$$
a(\operatorname{sen} \pi t,-\cos \pi t), \quad \alpha \geq 0
$$

de suluçós 2-periódicas, cujas órbitas cobrem todo o plano $x, y$. Além disso, para a $>0$ cssas soluçós's săo contra-fluxo.

Como antecipamos no início da seção, podemos construir ainda una família a tun parànetro de soluçoes que também cobrem o plano, que giram cm torno da origem no sentido horário.

De fato, considerando uma funça continua $\left.\varphi-\left(\varphi_{1}, \varphi_{2}\right): \vdots-1,0\right] \rightarrow \mathbb{R}^{2}$ : que satisfaz: $\varphi_{1}$ e $\varphi_{2}$ são cotritamente crescentes, $\varphi \in Q_{1}, \varphi_{1}(-1)=0$ o $\varphi_{2}(-1)>0$. Seja $z(t)=(x(t), y(t))$ soluçäo do (4.1) tal que $\left.z(t)\right|_{i-1.0]}=\varphi$.

Afrmanos que existe um primeiro instante $t_{1}>0$ em que $z(t)$ cruka o cixo $x$ com abcissa positiva.

De fato, se não existir tal $l_{1}$ definamos $(c, d)=z(1) \in Q_{1}$. Observando que

$$
\dot{y}(1) \leq-\pi \varphi_{1}(0)=-\eta<0
$$

para $t \geq 1$, e por

$$
y(t)-y(1) \leq-\eta(t-1)
$$


vê-se que $y(t)<0$ quamelo $t>{ }_{n}^{d}+1$.

Porlanto está demonstrado a nossa afirmaģão.

Tomando $\ell(t)-z_{1-1}$ podemos argumentar de forma análoga para garantir a existencia de $u m$ primeiro instante t.2 tal que $z(t)$ cruza o eixo y com ordenada loegativa.

Procedendo assim sucessivamente construímos uma solugão z $(t)$ que gira em torno da origem no sentido horário, conforme a tigura abaixo.

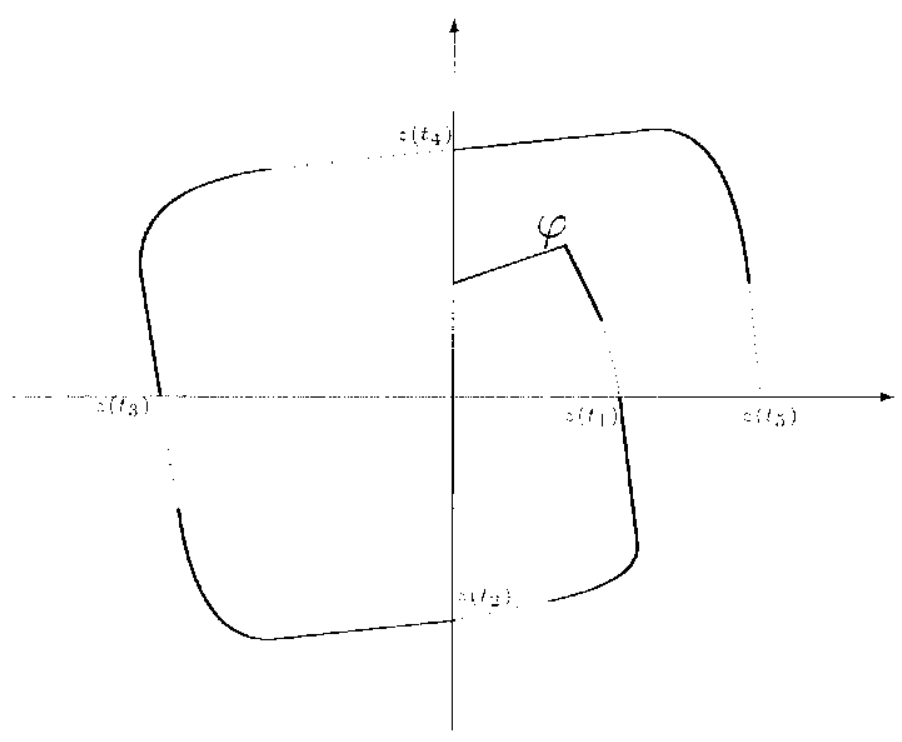

Figura 4.2: Sentido da órbita

Considerando que (1.1) é linear, $z(t)$ gera a família

$$
\alpha z(t): \quad \alpha>0
$$

(que cobre o planos $x, y$ e para $\alpha>0$ gira no senticlo horário.

A aparente contradição de o sistema (4.1) possuir duas famílias de soluções ambas cobrindo o plano $x, y$, uma girando no sentido oposto da outra, estaria na idéia ingenua de se entender as órbitas de (4.1) como as órbitas de um sistema 
dinámico bidimensional. É claro que o sistema dinámico definido por (4.1) tem dimensào infinitid.

\subsection{Estudo de um problema impulsivo}

As consideraçòes da seça anterior nos motivam a retomar o contexto mais geral da equação (3.1) e formular a seguinte questão: sob a hipótese (H1), que condiçoes deve satisfazer o sistema não-lineal

$$
\begin{aligned}
& \dot{x}_{1}(t)=F_{1}(x(t-1)) \\
& \dot{x}_{2}(t)=F_{2}(x(t-1))
\end{aligned}
$$

para que existam soluçöes contra-fluxo? E soluçōes periódicas contra-fluxo?

Mostraremos que é possível introdızir impulsos radiais de modo a garantir a existéncia de soluçòes contra-fluxo. Com alguma restrição ao segundo membro de (4.3) vanos garantir a existencia de soluçóes contra-fluxo perióclicas.

Lma análise cuidadosa dos fatos apresentados na seção 4.1 mostra que a construçäo de soluçoes contra-fluxo de (4.1) depende, de forma crucial, de que a soluçào $(x(t), y(t))$ satisfaça

$$
\begin{aligned}
& (x(t), y(t)) \in Q_{1} \Rightarrow(x(t-1), y(t-1)) \in Q_{3}, \\
& (x(t), y(t)) \in Q_{2} \rightarrow(x(t-1), y(t-1)) \in Q_{4}, \\
& (x(t), y(t)) \in Q_{3} \Rightarrow(x(t-1), y(t-1)) \in Q_{1}, \\
& (x(t), y(t)) \in Q_{4} \rightarrow(x(t-1), y(t-1)) \in Q_{2} .
\end{aligned}
$$

Fstas condiçoes acarretam que se $(x(t), y(t))$ está sobre un dos cixos cntão $(x(t-1), y(t-1))$ está sobre o messno eixo om posiçaio oposta com respeito à origem. 
As condiçós (4.4) são muito rígidas o se constituent, de fato, numa dificuldade extremat para se obter mma resposta positiva à nossa questäo.

No entanto, podemos acrescentar uma condição de auto-sustentação innulsiva ao sistema (1.3) do modo que certas soluçōes do sistema gozem das propriedades (4.1).

É importante observar que os impulsos serão radiais, isto ć, se t for um instante de impulso de uma solujão $x(t)=\left(x_{1}(t), x_{2}(t)\right)$ então $x(t+)$ c $x(t-)$ estarão alinhados com a origem. Portanto, a condição de auto-sustentação não afetará a orientação do campo em $\mathbb{R}^{2}$.

Nestas condiçoos provaremos que o sistema (4.3) acrescido de uma tal condição de anto-sustentação tem soluçăo contra-fluxo.

Vamos necessitale da seguinte definigälo.

Definição 4.2.1 Dada una curva

$$
\gamma(t)=\left(\gamma_{1}(t), \gamma_{2}(t)\right), \quad t \in[\alpha, \beta]
$$

do plano $x_{1}, x_{2}$; diz-se que $t \in(\alpha, \beta]$ é um instante crítico se $\gamma$ cruza lransversalmente um dos exos coordenados pela esquerda em $\gamma(b)$. Isto é, vale uma das allemativas:

$$
\begin{array}{ll}
\text { (i) } \gamma_{1}(t)=0, & \dot{\gamma}_{1}(t) \neq 0, \\
\text { (ii) } \gamma_{2}(t)=0 . & \dot{\gamma}_{2}(t) \neq 0 .
\end{array}
$$

Suponhamos que $F_{1}, \Gamma_{2}: \mathbb{R}^{2} \rightarrow \mathbb{R}$ sejam contínuas e satisfazem as seguintes hipóteses:

(H1) $x_{2} F_{1}(x)>0$, se $x_{2} \neq 0$ e $x_{1} F_{2}(x)<0$, se $x_{1} \neq 0$. 
(H2) Existe uma constante $M>0$ tal que $\left|F_{j}(x)\right| \leq M$ para todo $x \in \mathbb{R}^{2}$ $\because j-1,2$.

Consideremos o problema dado pelo sistema (4.3), onde $F^{\prime}(x)=\left(F_{1}(x), F_{2}(x)\right)$, sujeito à condição de impulso

$$
\begin{aligned}
& x_{1}(t)=0 \quad \text { e } \quad x_{2}(t-1) \neq 0 \rightarrow x_{2}(t+)--\int_{t-1}^{l-\frac{1}{2}} F_{2}(x(s)) d s, \\
& x_{2}(t)=0 \quad \text { e } \quad x_{1}(t-1) \neq 0 \Rightarrow x_{1}(t+)=-\int_{t-1}^{l-\frac{1}{2}} F_{1}(x(s)) d s
\end{aligned}
$$

para $t>0$.

Se $x(t) \neq(0,0)$, a notação $x(t+)$ significa

$$
x(t+)= \begin{cases}\left(x_{1}(t+), x_{2}(l)\right) . & \text { so } x_{1}(t)=0, x_{2}(t-1) \neq 0 \\ \left(x_{1}(t), x_{2}(t+)\right), & \text { se } x_{2}(t)=0, x_{1}(t-1) \neq 0\end{cases}
$$

Por (4.5) podemos notiar que o espaco $C=C\left(\left[-1.0, \mathbb{R}^{2}\right)\right.$ näo pode ser utilizado como espago de fases, neste caso consideraremos am espaço mais geral, dado por $G=G\left([-1,0], \mathbb{R}^{2}\right)$ que é o espaço das funçôes regradas, definidas em $[-1,0]$ com imagem em $\mathbb{R}^{2}$, cujas propriedades estào descritas no Capítulo 1.

Consicleremos o subconjunto $K$, do espago $G$, tal que $\varphi=\left(\varphi_{1}, \varphi_{2}\right) \in K$ so, a somente se.

(i) $\varphi_{1}(0)=\varphi_{1}(-1)=0, \varphi_{1}(0)$ é não decrescente para $\theta \in[-1,-1 / 2]$ é é não crescente para $\theta \in[-1 / 2,0] ;\left.\varphi_{1}\right|_{-1,0 \mid \backslash\left\{-\frac{1}{2}\right\}}$ é contínua.

(ii) $\varphi_{2}(-1 / 2)=0$ o $\varphi_{2}(0)$ é não decrescute para $\theta \in[-1,0] ; \varphi_{-1}$ 1, ó contínula.

(iii) $\left\|\varphi_{j}\right\| \leq M$ e $\varphi_{j}$ são $M$-Lipschitzianas, para $j=1,2$. 
Vamos supor que

$$
x_{0}-\varphi \in K
$$

Vamos definir solução para o problema impulsivo (4.3), (4.5) e (4.7), observando que os nomentos de impulso da solução devem ser instantes críticos, de acordo con in definiçào (4.2.1).

Definição 4.2.2 Uma soluşào do problema (4.8), (4.5) e (4.7) é uma funçäo $x=\left(x_{1}, x_{2}\right):[-1, w) \rightarrow \mathbb{R}^{2}, w>0$, satisfazendo:

(i) $\left.x\right|_{[-1,0]}=\varphi$

(ii) Lembrando que $F\left(x_{1}, x_{2}\right)=\left(F_{1}(x), F_{2}(x)\right)$,

$$
x(t)=\varphi(0)+\int_{0}^{t} F(\varphi(s-1)) d s, \quad t \in[0, \tau],
$$

onde $\tau=1$ ou $\tau \in(0,1)$ é o primeiro instante critico de $x(t)$.

Se $\tau$ é um instante crítico, cutâo

$$
x(t)=x(\tau+)+\int_{\tau}^{t} F(x(s-1)) d s, \quad t \in\left(\tau, \tau_{1}\right] \subset[0, w)
$$

onde $\tau_{1}=\tau+1$ ou $\tau_{1} \in(\tau, \tau+1)$ o primeno instante critico de $x(t)$ neste intervalo.

E ussim sucessivamente.

Vale observar que dada uma condição inicial no conjunto $K$, o problema (4.3),

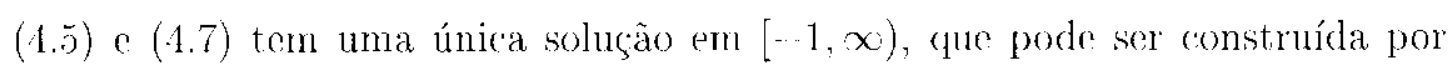
processo recursivo en sucessivos intervalos de comprimento unitário. Notemos 
que fixar $t_{0}=0$ năo constitui perda de gencralidade, uma vez que o problema é autonomo.

Tonando $\epsilon>0$ pequeno, podemes delinir a funçào $c(t)=\left(c_{1}(t), c_{2}(t)\right)$, para todo $t \in \mathbb{R}$ do seguinte nodo:

$$
\begin{aligned}
& c_{1}(t)= \begin{cases}2 \epsilon(t+1) . & \text { se } t \in[-1,-1 / 2] \\
-2 \epsilon t, & \text { se } t \in[-1 / 2,1 / 2\} \\
2 \epsilon(t-1) . & \text { se } t \subset[1 / 2,1] .\end{cases} \\
& c_{2}(t)= \begin{cases}2 \epsilon t+\epsilon & \text { se } t \in[-1,0] \\
-2 \epsilon(t-1 / 2), & \text { se } l \in[0,1]\end{cases}
\end{aligned}
$$

Estendemos $c(t)$ a toda a reta, de modo que ela seja 2-periódica. Notemos que $\left.c(t)\right|_{[-1, u]} \in K^{\prime}$.

Comsideremos agora o conjunto $K_{c} \subset K^{\circ}$ tal que $\varphi=\left(\varphi_{1}, \varphi_{2}\right) \in K_{\mathrm{c}}$ se $\varphi \in K$ ( so

$$
\begin{aligned}
\varphi_{1}(\theta) \geq c_{1}(\theta), & \theta \in[-1,0] \\
\left|\varphi_{2}(\theta)\right| \geq\left|c_{2}(\theta)\right| . & \theta \subset[1,0] .
\end{aligned}
$$

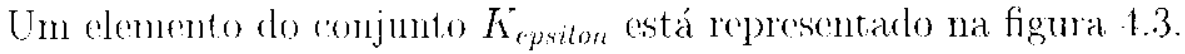

Observação 4.2.1 A funçào F satisfaz a hipótese (H1) que também está satisfeita no capitulo 3. Naquele cuso, esla hipótese força as órbitas das soluçoes a. 


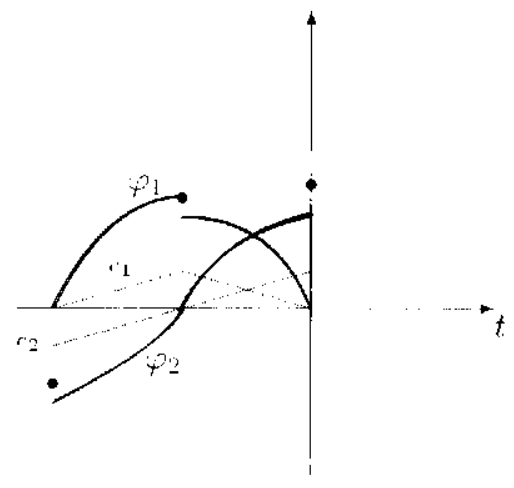

Figura 4.3: $--\left(\varphi_{1}, \varphi_{2}\right) \in K_{\ell}$

girar no sentido horário em torno da origem. Aqui, entretanto, dado $\varphi \in K_{\text {, }}$ se denotarmos $\bar{\varphi} \in K_{\epsilon}$ definida por

$$
\bar{\varphi}(\theta)=\varphi(\theta) \quad \text { para }-1 \leq \theta<0 \quad \text { e } \quad \varphi(0)=\left(0,-\int_{-1}^{-1 / 2} F_{2}(\varphi(s)) d s\right)
$$

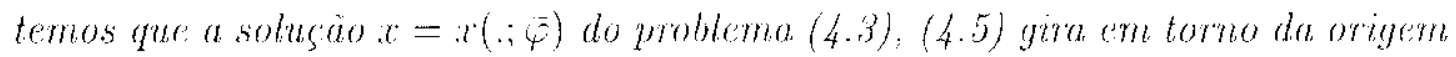
no sculde anti-horimio, conforme a figura 4.4 .

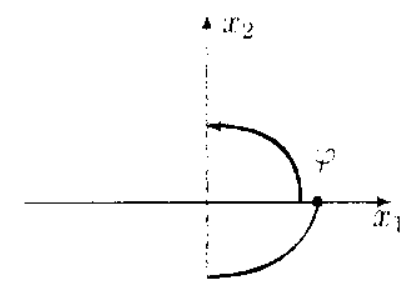

Figura 4.4: Sentido das órbitas de $x(. ; \bar{\varphi})$ no plano $x_{1} x_{2}$.

Sejam $f_{1}: \mathbb{R} \rightarrow \mathbb{R}: \int_{2}: \mathbb{R} \rightarrow \mathbb{R}$ funçoes coutínuas e crescentes, que satisfazem as seguintes hipóteses 

(A) $s \int_{j}(s)>0, \quad$ se $s \neq 0$ e, $j=1,2$;
(B) $\Xi M>0 ; \quad\left|f_{j}(s)\right| \leq M, \quad \forall s, \quad j=1,2 ;$
(C) $\left|f_{j}(s)\right| \geq 4|s|$ para $|s|<\delta, \delta>0$ pequeno e $j=1,2$.

A condição (A) garante (que para $F\left(x_{1}, x_{2}\right)=\left(f_{1}\left(x_{2}\right),-f_{2}\left(x_{1}\right)\right)$, a hipótese (H1) está satisfeita.

Consideremos o problema dado pelo sistema

$$
\begin{aligned}
& \dot{x}_{1}(t)=f_{1}\left(x_{2}(t-1)\right) \\
& \dot{x}_{2}(t)=-f_{2}\left(x_{1}(t-1)\right)
\end{aligned}
$$

sujeito à condiça de impulso

$$
\begin{aligned}
& x_{1}(t)=0 \quad \text { e } \quad x_{2}(t-1) \neq 0 \Rightarrow x_{2}(t+)=\int_{1}^{t} f_{2}^{t} f_{2}\left(x_{1}(s)\right) d s, \\
& x_{2}(t)=0 \quad \text { c } \quad x_{1}(t-1) \neq 0 \Rightarrow x_{1}(t+)--\int_{l-1}^{t-\frac{1}{2}} f_{1}\left(x_{2}(s)\right) d s
\end{aligned}
$$

para $t>0$.

Se fizermos $e=\delta$ e consideramos o conjunto $K_{\delta}$ : pela observaçào 4.2 .1 , o problema (4.8) e (4.9) com a condiçăo inicial cm $K_{\delta}$, tem solusão contra-fluxo. Em seguida mostraremos que existe soluçào contra-fluxo desse problema que é periódica.

Por arlaptaçoes diretas de argmunentos clássicos de compacidade no esparco $C\left(I, \mathbb{R}^{n}\right)$ (I um intervalo compacto) pode-se provar que $K_{\delta}$ é comparcto co convexo. Assim, basta definirmos um operador de retorno contínuo de $K_{\delta} \mathrm{cm} K_{\delta}$ : cujos pontos fixos corresponderão a soluções periódicas.

Definamos

$$
A: h_{\delta} \rightarrow G \quad \text { por } \quad A(\varphi)=x_{2}(., \bar{\varphi})
$$


onde $\bar{\varphi}$ edada por

$$
\bar{\varphi}(\theta)-\varphi(\theta) \quad \text { para } \cdots 1 \leq \theta<0 \quad \text { e } \varphi(0)=\left(0, \int_{-1}^{-1 / 2} f_{2}\left(\varphi_{1}(s)\right) d s\right) .
$$

Eur $(1.10), x_{2}(\therefore \bar{\varphi})$ representa $x_{T}(. ; \bar{\varphi})$ com $\tau=2$ o não a segunda coordenada de $x(. ; \bar{\varphi})$.

Teorema 4.2.1 O operador $A$ definido em (4.10) é contínuo e $A\left(K_{\delta}\right) \subset K_{\delta}$.

Demonstração: Seja $\varphi \in K_{\delta}$. Vamos mostrar que $A \varphi \in K_{\delta}$ por construção.

Primciramente, consideremos $t \in[0,1 / 2]$ e $\bar{\varphi}$ como na observação (4.2.1).

Pelas hipóteses sobre a $f_{2}$, podemos afirmar que

$$
\begin{aligned}
x_{2}(0) & \left.=\int_{1}^{1 / 2} f_{2}\left(\varphi_{1}(s)\right) d s \geq \int_{1}^{1 / 2} f_{2}\left(c_{1}(s)\right) d s \geq 4 \int_{-1}^{-1 / 2} c_{1}(s)\right) d s=\delta \\
f_{2}\left(\varphi_{1}(s)\right) & \geq 0 \text { c ce crescente para } s \in-1,-1 / 2], \text { portanto } \\
x_{2}(t ; \varphi) & =x_{2}(t)=x_{2}(0)-\int_{0}^{t} f_{2}\left(\varphi_{1}(s-1)\right) d s \\
& -\int_{-1}^{-1 / 2} f_{2}\left(\varphi_{1}(s)\right) d s-\int_{-1}^{t-1} f_{2}\left(\varphi_{1}(s)\right) d s \quad t \in[0,1 / 2]
\end{aligned}
$$

é decrescente no intervalo $[0,1 / 2]$ e $x_{2}(t)>0$ para $t$ em $[0,1 / 2)$, satisfazendo $x_{2}(1 / 2)=0$. Alén disso, $x_{2}(t)$ é uma função côncava en $[0,1 / 2]$, o que inplica.

$$
\left|x_{2}(t)\right| \geq\left|c_{2}(t)\right|: \quad t \in[0,1 / 2 \vdots
$$

No caso da coordenada $x_{1}$, temos que $f_{1}\left(\varphi_{2}(s)\right) \leq 0$ e é crescente para $s \in$ $-1,-1 / 2 ;$, nntão $x_{1}(t)$ dado por

$$
x_{1}(t: \varphi)=x_{1}(t)=\int_{0}^{t} f_{1}\left(\bar{\varphi}_{2}(s-1)\right) d s=\int_{-1}^{t} f_{1}\left(\varphi_{2}(s)\right) d s \quad t \in[0,1 / 2]
$$


é decrescente e negativa em $[0,1 / 2] \mathrm{e}$

$$
x_{1}(1 / 2)=\int_{1}^{\cdots 1 / 2} f_{1}\left(\varphi_{2}(s)\right) d s \leq \int_{1}^{\cdots 1 / 2} f_{1}\left(c_{2}(s)\right) d s \leq 1 \int_{-1}^{1 / 2} c_{2}(s) d s=\cdots \delta .
$$

Além disso, li (l) ó una funçáo convexa en $0,1 / 2]$, logo está satisfeita a condiçà̃o

$$
\left|x_{1}(t)\right| \geq\left|c_{1}(t)\right|, \quad t \in[0,1 / 2]
$$

Observemos que a coordenada $x_{1}(t)$ sofre impulso em $t=\frac{1}{2}$, já que $x_{2}(1 / 2)=0$ e $x_{1}(-1 / 2)=\varphi_{1}(-1 / 2) \neq 0$, que satislaz

$$
\begin{aligned}
x_{1}(1 / 2+)=-\int_{1 / 2}^{0} f_{1}\left(x_{2}(s)\right) d s & =-\int_{-1 / 2}^{0} f_{1}\left(\varphi_{2}(s)\right) d s \\
& \leq-\int_{-1 / 2}^{0} f_{1}\left(c_{2}(s)\right) d s \leq-1 \int_{-1 / 2}^{0} c_{2}(s) d s=-\delta .
\end{aligned}
$$

Como $f_{1}\left(\varphi_{2}(s)\right) \geq 0$ o c crescente para $s \in(-1 / 2.0]$, vale que $x_{1}(t)$ dado por

$$
x_{1}(t)=x_{1}(1 / 2+)+\int_{1 / 2}^{t} \int_{1}\left(\varphi_{2}(s-1)\right) d s \quad t \in[1 / 2,1]
$$

é cresconte o negativa no intervalo $\left(1 / 2,1\right.$. Fm $t=1, x_{1}(t)=0$ e $x_{2}(t-1)=$ $x_{2}(0) \neq 0$ e portanto $l=1$ é um instante de impulso. Além disso, $x_{1}(t)$ é uma função convexa neste intervalo, logo

$$
\left|x_{1}(t)_{i} \geq\right| c_{1}(l), \quad t \in[1 / 2,1] .
$$


$x_{2}(1 / 2)=0, f_{2}\left(\varphi_{1}(s) \geq 0\right.$ e c decrescente para $s \in(-1 / 2,0]$, isso tudo implica que $x_{2}(t) 6$ decrescente e negativa no intervalo $[1 / 2,1$. Para $t=1$ vale

$$
x_{2}(1)=-\int_{-1 / 2}^{0} f_{2}\left(\varphi_{1}(s)\right) d s \leq 4 \int_{-1 / 2}^{\cdots 0} c_{1}(s) d s=-\delta,
$$

Observando tantbém çue $x_{2}(1)$ á una função convexa em $[1 / 2,1]$, podemos conchuir que

$$
\left|x_{2}(t)\right| \geq \mid c_{2}(t)^{1}, \quad t \in[1 / 2,1] .
$$

Agora, para $t \in[1,3 / 2]$, temos que $x_{2}(t)$ sofre impulso em $t=1$, e satisfa

$$
x_{2}(1+)=\int_{0}^{1 / 2} f_{2}\left(x_{1}(s)\right) d s \leq 4 \int_{0}^{1 / 2} c_{1}(s) d s=-\delta
$$

Como $f_{2}\left(x_{1}(s)\right) \leq 0$ é decrescente para $s \in[0,1 / 2]$, então $x_{2}(t)$ dado por

$$
x_{2}(l)=x_{2}(1+)-\int_{1}^{l} f_{2}\left(x_{1}(s-1)\right) d s \quad l \in(1,3 / 2]
$$

ć crescente e negativa para $t \in(1,3 / 2] . \operatorname{Em} t=3 / 2, x_{2}(t)=0$ e $x_{1}(3 / 2-1)=$ $x_{1}(1 / 2) \neq 0$. O que nos diz que $t=3 / 2$ é um instante de impulso. Notemos também que $x_{2}(t)$ é uma função convexa no intervalo $(1,3 / 2]$, logo

$$
\left|x_{2}(t)\right| \geq \mid c_{2}(t) i, \quad t \in[1,3 / 2]
$$

Como $x_{1}(1)=0$ e $f_{1}\left(x_{2}(s)\right) \geq 0$ para $s \in[0,1 / 2]$, então $x_{1}(t)$ dado por

$$
x_{1}(t)-\int_{1}^{t} f_{1}\left(x_{2}(s-1)\right) d s \quad t \in[1,3 / 2]
$$

ć crescente e positiva neste intervalo. Para $t=3 / 2$, vale 


$$
x_{1}(3 / 2)=\int_{1}^{3 / 2} f_{1}\left(x_{2}(s-1)\right) d s \geq \int_{0}^{1 / 2} f_{1}\left(c_{2}(s)\right) d s \geq 4 \int_{0}^{1 / 2} c_{2}(s) d s=\delta
$$

Além disso, $x_{1}(t)$ é uma função concava en $\left[1,3 / 2 ;\right.$ pois $\dot{x}_{1}(t)=f_{1}\left(x_{2}(t-1)\right)$ s decrescente. Portanto vale

$$
\left|x_{1}(l)\right| \geq\left|r_{1}(l)\right|: \quad t \subset[1,3 / 2]
$$

Finalmente para $t \in[3 / 2,2], x_{1}(t)$ sofre inpulso em $t=\frac{3}{2}$, e satisfaz

$$
x_{1}(3 / 2+)=-\int_{1 / 2}^{1} f_{1}\left(x_{2}(s)\right) d s \geq-\int_{1 / 2}^{1} f_{1}\left(c_{2}(s)\right) d s \geq 4 \int_{1 / 2}^{1} c_{2}(s) d s=\delta .
$$

Como $f_{1}\left(x_{2}(s)\right) \leq 0$ é decrescente para $s \in[1 / 2,1]$, cntano $x_{1}(l)$ dada por

$$
x_{1}(l)-x_{1}(3 / 2+)+\int_{3 / 2}^{l} f_{1}\left(x_{2}(s-1)\right) d s, \quad t \in[3 / 2,2]
$$

é decrescente positiva no intervalo $\left(3 / 2,2\right.$. Notemos que $x_{1}(2)=0$ e $x_{2}(2-1) \neq$ 0 . Além disso, $x_{1}(t)$ é uma função côncava em $(3 / 2,2]$, e portanto

$$
\left|x_{1}(t)\right| \geq\left|c_{1}(t)\right|, \quad t \in[3 / 2,2]
$$

$$
x_{2}(3 / 2)=0 \text { c como } f_{2}\left(x_{1}(s)\right) \leq 0 \text { para } s \in(1 / 2,1] \text {, wata } x_{2}(t) \text { é crescente } \theta
$$
positiva no intervalo $(3 / 2,2]$. Para $t=2$ vale

$$
\left.x_{2}(2)=-\int_{3 / 2}^{2} f_{2}\left(x_{1}(s-1)\right) d s \geq-\int_{1 / 2}^{1} f_{2}\left(c_{1}(s)\right) d s \geq-4 \int_{1 / 2}^{1} c_{1}(s)\right) d s=\delta
$$


ou seja, $x_{2}(2) \geq \delta$. Notemos que $x_{2}(l)$ é unla funçăo côncava neste intervalo, pois $\dot{x}_{2}(t)=-f_{2}(x(t-1))$ é decrescente, $\log 0$

$$
\left|x_{2}(t)\right| \geq \mid c_{2}(t) !, \quad t \in[3 / 2,2]
$$

Provarlo isto, fica claro que $x_{\tau}(; \bar{\varphi}) \in K_{\delta}$ com $\tau=2$. Portanto $A K_{\delta} \subset \Lambda_{\delta}$.

Seja agora $\varphi^{n}=\left(\varphi_{1}^{n}, \varphi_{2}^{n}\right) \in K_{\delta}, n=1,2, \cdots$. Se $\varphi^{n} \rightarrow \varphi=\left(\varphi_{1}, \varphi_{2}\right)$ quando $n \rightarrow \infty$, então $x\left(t: \bar{\varphi}^{n}\right) \rightarrow x(t ; \bar{\varphi})$. para $t \in[0,1 / 2]$ uniformemente, ou seja,

$$
x_{1 / 2}\left(\cdot ; \bar{\varphi}^{n}\right) \rightarrow x_{1 / 2}(\cdot \bar{\varphi}) . \quad n \rightarrow \infty
$$

Tomando $\psi^{n}-x_{1 / 2}\left(\cdot ; \bar{\varphi}^{n}\right) e \psi-x_{1 / 2}(\cdot \dot{\varphi})$, com

$$
\psi_{1}^{n}(1 / 2+)=-\int_{1 / 2}^{0} f_{1}\left(\psi_{2}^{n}(s)\right) d s, \quad \psi_{1}(1 / 2+)=-\int_{-1 / 2}^{0} f_{1}\left(\psi_{2}(s)\right) d s
$$

vê-se analogamente (que; $x_{1}\left(. ; \bar{\varphi}^{n}\right) \rightarrow x_{1}(. ; \phi)$ quando $n \rightarrow \infty$. Aqui também $x_{1}(; \bar{\varphi})$ indica $x_{\tau}(; \bar{\varphi})$ lal que $\tau=1$.

Procedendo assim mais duas vezes sucessivas. verificamos que

$$
x_{2}\left(. \varphi^{\prime \prime}\right) \rightarrow x_{2}(. \bar{\varphi}) \quad \text { quando } n \rightarrow \infty
$$

Portanto a o operador de retorno $A$ é contínuo.

Pelo Teorema de Schauder, existe um ponto fixo de 1 o que implica que existe uma solução 2-periódica contra-fluxo de (4.8) e (4.9). 


\section{Referências Bibliográficas}

[1] M.Z. Baptistini and P.Z. Táboas. On the existence and global bifurcation of periodic: solutions to planar differential delay equations, Jomrmal of Differential Equations, Vol. 127, 391-425 (1996).

[2] S.M.S. Godoy, J.G. Reris, Stability and existence of periodic solutions of a functional-differential equation, Joumal of Mathematical Analysis and $\Lambda$ pplications, vol. 198, 381-398 (1996).

[3] K. Gopalsamy, B.G. Zhang, On delay differential equations with impulses, Journal of Mathematical Analysis and Applications, vol. 139, 110-122 (1989).

[4] J.K. Hale and S.M.V. Imnel, Introduction to Functional Differential Equations, New York, Springer $(1993)$.

[5] Chaim S. Hönig, Volterra Stieltjes-Integral Equations, North-Holland Publishing Company (1975).

[6] E. Kruger-Thimer, Formal theory of drug dosage regiments, I. Joumal Theo. Biology, Vol. 13 (1966).

[7] V. Lakshmikantham. D.D. Bainor c P.S. Simeonov, Theory of Impulsive Differential Equations, World Scjentific Publishing Company, Singapore (1989).

[8] B. Li and Y. Kuang, Sharp conditions for oscillations in some nonlincar nonantonomous delay differntial equations, Nonlinear analysis, theory, methods and applications, vol. 29, n $11,1265-1276$ (1997). 
[9] H. Matsunaga, R. Miyazaki, 'I'. Lara, Global at tractivity results for nonlinear delay differential equations, Journal of mathematical analysis and applications, vol. 234, 77-90)(1999).

[10] A.D. Myshkis, Autonomous differential equations with impulsive self-support and infinite delay, Functional Differential lequations, Vol. 3, n' 1-2, 145-154 (1995).

[11] P.Z. Táboas, Periodic solutions of a planar delay equations, Proc. Royal Society Edinburgh, Vol. 116A, 85-101 (1990).

[12] M.E. Quiroga, Sobre alguns problemas de periodicidade em equaçoes diferenciais impulsivas com retardamento, Tese de Doutorado, Universidade de Sào Paulo (1999).

[13] Y. Zhang, A. Zhao, J. Yan, Oscillation criteria for impulsive delay differential equations, Journal of Mathematical Analysis and Applications, vol. 205, 461$470(1997)$.

[14] A. Zhao, J. Yan, Asymptotic behavior of solutions of impulsive delay differential equations, Journal Mathematical Analysis and applications, vol, 201, $943-954(1996)$.

[15] A. D. Myshkis, Auto-oscillations in continuos systems with impulsive solfsupport, em Resenhas do IME-LSP, vol.3, n 1, 93-106 (1997). 